\title{
Modeling inter-human movement coordination: synchronization governs joint task dynamics
}

\author{
Alexander Mörtl Tamara Lorenz Björn N.S. Vlaskamp \\ Azwirman Gusrialdi Anna Schubö Sandra Hirche \\ Draft
}

\begin{abstract}
Human interaction partners tend to synchronize their movements during repetitive actions such as walking. Research of inter-human coordination in purely rhythmic action tasks reveals that the observed patterns of interaction are dominated by synchronization effects. Initiated by our finding that human dyads synchronize their arm movements even in a goal-directed action task, we present a step-wise approach to a model of inter-human movement coordination. In an experiment, the hand trajectories of ten human dyads are recorded. Governed by a dynamical process of phase synchronization, the participants establish in-phase as well as anti-phase relations. The emerging relations are successfully reproduced by the attractor dynamics of coupled phase oscillators inspired by the Kuramoto model. Three different methods on transforming the motion trajectories into instantaneous phases are investigated and their influence on the model fit to the experimental data is evaluated. System identification technique allows us to estimate the model parameters, which are the coupling strength and the frequency detuning among the dyad. The stability properties of the identified model match the relations observed in the experimental data. In short, our model predicts the dynamics of inter-human movement coordination. It can directly be implemented to enrich human-robot interaction.
\end{abstract}

\section{Introduction}

People coordinate their movements in many situations of daily life. This movement coordination can be intrapersonal, e.g. coordination of one's left with one's right arm or interpersonal, i.e. coordination with another person. There is a wide range of actions that people can do together and for all these actions movements need to be coordinated. Examples range from handing over objects, manipulating a common workpiece to setting up a table. In order to prevent collisions and injuries in the worst case, the agents are required to keep certain spatial and temporal relations of their actions. People often seem to achieve such coordination without much effort in a smooth manner. 


\subsection{Synchronization - a ubiquitous feature of human movement coordination}

Coordination often comes about as movement synchronization during repetitive action. Synchronization can be understood as the establishment of a bounded temporal relationship between the interacting entities, which is brought about and sustained by a convergent dynamical process. Many examples in literature provide evidence for movement synchronization. When walking in a group, people tend to synchronize their gait [van Ulzen et al., 2008]. In experiments requiring relatively simple movements, synchronization is found to be a stable pattern in human behavior. Richardson et al. [2007] introduce a paradigm in which two people are moving next to each other in rocking chairs. They unintentionally rock in synchrony, although different weights attached to the chairs manipulate the frequencies at which they would naturally oscillate without human effort. Similar behavior is observed in tasks in which people have to swing handheld pendulums [Richardson et al., 2005] or are merely moving their legs [Schmidt et al., 1990]. Besides these behavioral effects, synchronization affects social relationships. It is found that falling into synchrony with somebody else serves a purpose: it enhances perceptual sensitivity towards each other, fosters cooperative abilities [Valdesolo et al., 2010] and leads to the attribution of more positive characteristics to the interaction partner [Miles et al., 2009]. All in all synchronization of movements seems to play an important role in human interactive behavior.

The contribution of this article is a systematic approach to describe human-human interaction $(H H I)$ in a quantitative way. Our goal is to provide a description that can be applied directly in human-robot interaction $(H R I)$ for its evaluation. We therefore address three research questions in this article: First, is movement synchronization in HHI a phenomenon that also holds in goal-directed tasks? Second, if yes, when does synchronization between humans emerge during such a task and which strategy is applied to do so? Third, how can we capture the observed effects in a mathematical model which is transferable to a robotic agent? The analysis of the experimental data gathered in our previous study [Lorenz et al., 2011] shows that people also synchronize their arm movements in a goal-directed task which is characterized by the need for precise movements. These findings are in line with previous studies on the topic [Schmidt et al., 1990, Richardson et al., 2005, 2007]. In general, movement synchronization is found to be a guiding dynamical process which leads to stable coordination patterns in natural HHI. With our approach, we characterize the emerging patterns and the quality of coordination by the extent of phase synchronization. Through system identification based on the experimental data we obtain a parameterized model which is ready for implementation and evaluation in HRI.

Since modeling of HHI is the central contribution of this article, an overview on related work towards the exploration and modeling of human movement coordination is provided in the following.

\subsection{Modeling rhythmic movement coordination}

Movement synchronization - as a basic principle of human interactive behavior - is investigated by means of dynamical systems that model interconnected perception-action 
loops and generate dynamical patterns [Schöner and Kelso, 1988, Beek et al., 1995b]. Warren [2006] provides a detailed introduction on behavioral dynamics in this context. Pioneering work on modeling intrapersonal coordination of limbs is conducted by Haken et al. [1985]. The authors propose a minimal dynamical model of coupled nonlinear oscillators - known as the Haken-Kelso-Bunz (HKB) model - which successfully reproduces the transition between stable coordination patterns during bimanual fingertapping when changing the cycle frequency as the control parameter. The observed change of attractor basins depending on the extrinsic control parameter is qualitatively described by a sinusoidal potential function.

Several modifications of the HKB model are proposed. Beek et al. [2002] introduce two additional oscillators to represent the level of movement generation through the end effectors while explicitly accounting for the mechanical properties of a human limb. This neuro-mechanical model should foster the fundamental analysis of the interplay between the neural and the effector level during purely rhythmic simple movements. In order to gain deeper insights into the dynamics of human coordination, experimental paradigms which utilize a human's periodical input to drive oscillatory mechanical systems with different eigenfrequencies are applied repeatedly. Schmidt et al. [1993] employ the task paradigm of swinging pendulums originally introduced by Turvey et al. [1986]. An extension of the HKB coupling function by a frequency detuning term similar to the coupling function proposed by Cohen et al. [1982] is found to account for both the effects of different eigenfrequencies and external forcing frequencies.

Originally developed to model intrapersonal coordination of movements, dynamical models of coupled oscillators are found to qualitatively explain interpersonal coordination as well. While participants had to swing hand-held pendulums, Schmidt and O'Brien [1997] show the emergence of unintended coordination in a laboratory task. It is argued that the same mechanisms of dynamical self-organization as observed in intrapersonal coordination apply. In a subsequent study, Schmidt et al. [1998] compare the characteristics of intended intra- and interpersonal coordination by applying the same pendulum paradigm. Frequency detuning imposed through different eigenfrequencies and frequency levels are introduced as control parameters. Depending on the frequency level and the intended phase relation, the authors obtain the coupling strength of a local dynamical model similar to Cohen et al. [1982]. The number of coordination breakdowns, the phase fluctuation and the coupling strength reveal interpersonal coordination to be weaker than intrapersonal coordination. However, the regression method to identify the coupling strength relies on a-priori knowledge of the pendulum-wrist system's frequency detuning, which limits the approach to oscillatory effector systems.

Fundamental research work on fitting nonlinear dynamic models to trajectories of human rhythmic movements is conducted by Kay et al. [1987]. Observed functional relationships between the external driving frequency and the amplitudes and peak velocities of the movements are found to be reproduced well by a mixture of van der Pol and Rayleigh oscillators with stable parameter fits. The model is fitted to the limit cycle data in the position-velocity phase plane. In a more general approach, Eisenhammer et al. [1991] propose a reconstruction method of time series data based on polynomial dynamical models which are fitted to the vector field of an appropriate state-space representation of the data. While also transients of a pair of coupled oscillators can be 
reconstructed from simulated data, the method is rather sensitive to noise and requires extensive observation of transient regimes to yield stable results, since the whole statespace region is reconstructed. Inspired by the numerous variations of coupled oscillators models of rhythmic limb movements, Beek et al. [1995a] systematically analyze how different components such as linear and nonlinear elastic and friction terms contribute to the composition of rhythmic movement. Jirsa and Kelso [2005] show in their work on dynamical movement models how the attractor landscape in its state space can be formed to reproduce a variety of both discrete and rhythmic movement behaviors, using their so-called excitators. Elementary human movement trajectories in response to different stimuli are replicated qualitatively by stimulating the respective excitator model. The authors show that their approach extends to coupled dynamical systems as well, yet given a certain action task it may require sophisticated design to obtain the desired features of coordinated movement behavior. The aforementioned approaches accurately model basic human motor behavior in rhythmic tasks, yet the movement reproduction and coordination is tightly encoded by the functional state-space representation of the oscillator dynamics, making them less flexible in their application to constrained, goal-directed tasks.

Similar to Beek et al. [2002], de Rugy et al. [2006] propose a neuro-mechanical unit per agent which employs a cross-coupled pair of self-sustained oscillators. The intended modes of coordination while swinging pendulums are replicated as well as the effect of resonance tuning when the pendulums were manipulated individually. Rocking side-by-side in chairs is introduced in a task paradigm involving whole-body movements by Richardson et al. [2007]. Analytical results on observed coordination patterns are also related qualitatively to the features of the HKB model, yet explicit modeling is not conducted.

Common to the fundamental research work on rhythmic movement coordination between humans, the exploration and modeling is mostly approached by means of combined oscillatory task-effector systems, such as hand-held pendulums. However, little is known about the coordination behavior of humans in more realistic action tasks, to what extent the fundamental findings and modeling approaches on movement coordination apply, and how the observed effects can be described in a quantitative way.

The remainder of this article is organized as follows: In Sect. 2 we review the design of our experimental task and outline the pursued modeling approach. The reader is provided with the methods used for data reduction and analysis in Sect. 3. Analytical findings, development of the model structure and the parameter identification are presented in Sect. 4. After discussing the results on dyadic movement coordination in Sect. 5 we draw our conclusions in Sect. 6.

\section{Approach}

In this section, a brief description of the experimental task is given. Movement data of the experiment also described in [Lorenz et al., 2011] are the basis for the investigation of our research questions. We further provide a sketch of our modeling approach including the mathematical definition of synchronization we refer to in this article. 


\subsection{Experimental task}

The investigation of our questions on human movement synchronization places certain requirements on the design of an experimental task. We consider the following points to be relevant for synchronization:

- The task paradigm should integrate goal-directed as well as repetitive actions which similarly appear in real-life settings. To keep things simple, the task is to execute a repetitive sequence consisting of a forward and a backward movement in a closed trajectory, which we call a cycle in the following. Multiple cycles are to be completed in a continuous manner, which allows synchronization to emerge among the agents. The forward as well as the backward movement (halfcycles) are point-to-point arm movements while carrying a tool in hand. This is where goal-directedness comes into play: the tool (a pen) has to be placed on two marked positions on a table alternately. Note that the agents perform identical tasks.

- The topology of workspaces is arranged without overlap of the movement trajectories. This enables the agents to perform in parallel without any interference or demand for collision avoidance, which could restrict movement synchronization.

- During task execution the agents' movements might be affected by sensory information that is available of the other agent's movements In order to provide full visual information, the agents are facing each other without any occlusions. Since they are always able to observe their opponents' movements as well as start and target positions, synchronization is made as easy as possible.

The setup of the laboratory task involving the actions of two agents is depicted in Fig. 1.

To investigate how movement synchronization is initialized, the action start off among the dyad is modulated by triggering it acoustically. Among all possible start off relations, three conditions are supposed to be capable of being differentiated in our experimental task. They are picked as follows: 1) both agents are triggered at the same time (zero-cycle). 2) the second agent is triggered when the first agent has passed a quarter of the first cycle, i.e. half the distance between start and target (quartercycle). 3) the second agent is triggered when the first agent has passed half of the first cycle, i.e. reached the target (half-cycle). After the performance of ten cycles a trial is completed and the agents are triggered to return to their start positions. For details on the experiment the reader is referred to the appendix.

\subsection{Synchronization of coupled dynamical systems}

Our goal is to capture the temporal relationship between the dyads' end effector motions in our experiment. To that extent we propose a modeling approach based on the synchronization of coupled dynamical systems. 


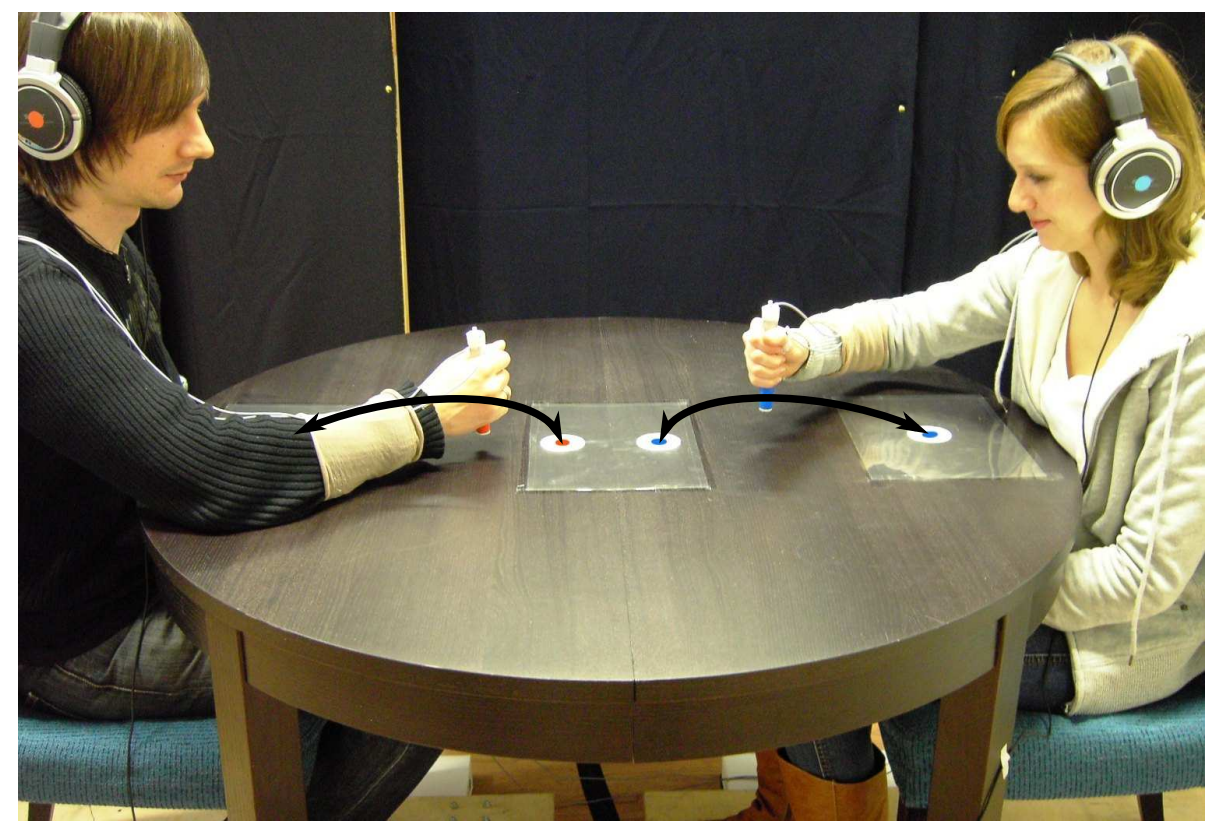

Figure 1: Experimental setup with two human agents. Participants are sitting face to face. The task is to alternately tap with a pen on two assigned points marked at the table. The action start off is triggered acoustically via headphones. Motion trajectories of the pens are captured by a visual tracking system

\subsubsection{Defining synchronization among coupled oscillators}

In this article, we adopt the dynamical systems approach to describe movement synchronization: the emerging coordination patterns are represented by the attractors of a dynamical system. Thereby attractors denote the regions in the dynamical system's state-space to which the system's trajectories are attracted. In particular, we treat the observed phenomena of movement coordination in an action task as a synchronization problem of two coupled oscillators forming a coupled dynamical system, which is in line with existing modeling approaches [Haken et al., 1985, Rand et al., 1988, Schmidt et al., 1993]. The general dynamical equations of two limit cycle oscillators that are mutually coupled are given by

$$
\begin{aligned}
& \dot{\xi}_{1}=F_{1}\left(\xi_{1}\right)+G_{1}\left(\xi_{1}, \xi_{2}\right) \\
& \dot{\xi}_{2}=F_{2}\left(\xi_{2}\right)+G_{2}\left(\xi_{2}, \xi_{1}\right),
\end{aligned}
$$

where $\xi_{i}$ is a vector of variables of any dimension for oscillator $i, F_{i}$ represents the limit cycle and $G_{i}$ is the coupling function that bidirectionally links the oscillators. If the oscillators are harmonic, we can simply write $F_{i}=\omega_{i}$ and $\xi_{i}=\theta_{i}$, with the oscillator's natural frequency $\omega_{i}$ and its phase $\theta_{i}$. Depending on the coupling function $G_{i}$, the 


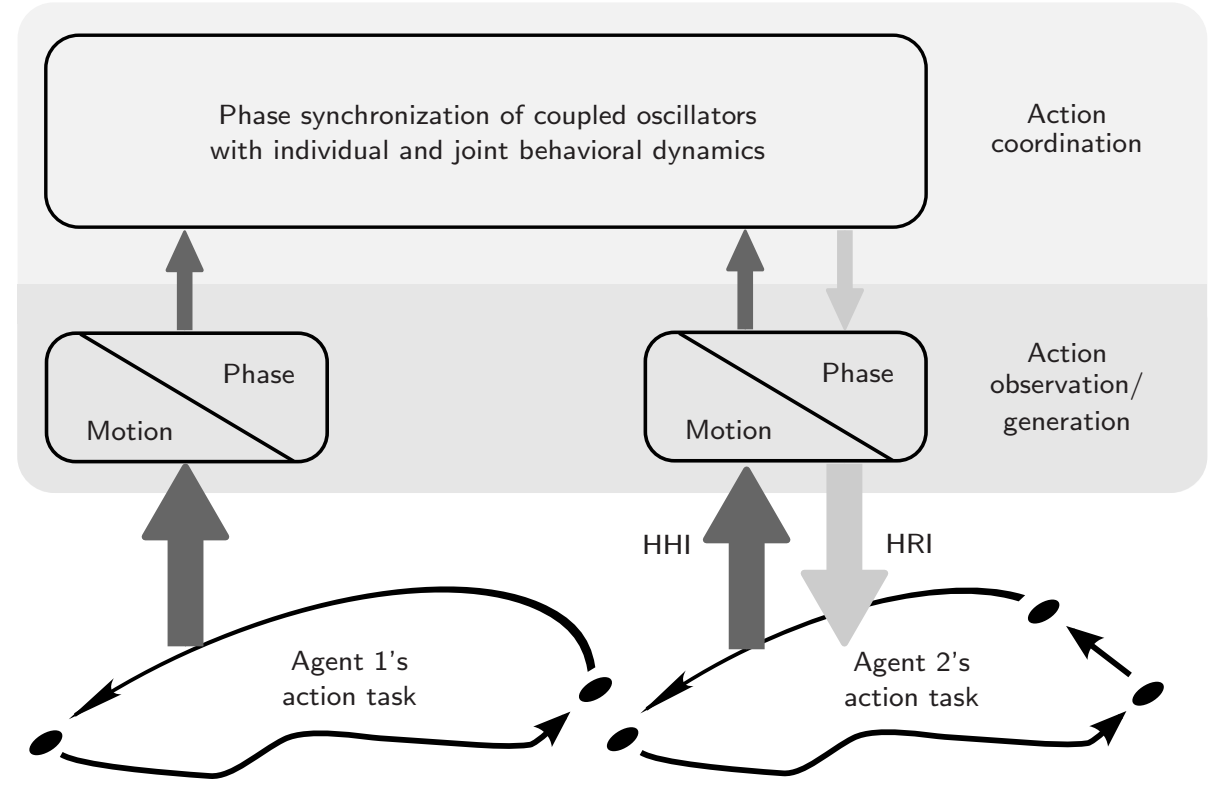

Figure 2: Overview scheme depicting the two-layered model of movement coordination. Agent 1 and 2 jointly engage in repetitive actions. The observed actions are transformed to phases in the lower layer. In the upper layer, the action coordination is governed by the individual and joint behavioral dynamics modeled as coupled phase oscillators. Dark arrows represent the modeling stage conducted in this article $(\mathrm{HHI})$. Light arrows outline the envisaged stage of action generation when the scheme is deployed to a robotic agent $2(\mathrm{HRI})$

oscillators may interact such, that

$$
\left|\Phi_{n, m}(t)\right|<\varepsilon, \text { with } \Phi_{n, m}(t)=n \theta_{1}(t)-m \theta_{2}(t)
$$

holds, with the generalized phase difference $\Phi_{n, m}(t)$ and a positive constant $\varepsilon \in \mathbb{R}$. Positive constants $n, m \in \mathbb{N}$ allow to detect synchronization of orders $n: m$. Thus, if $\Phi_{n, m}(t)$ becomes constant or fluctuates within some bounds, $F_{i}$ are synchronized, which is also called phase locking [Pikovsky et al., 2001].

Note: Since $\Phi_{n, m}(t)$ quantifies the interaction in a single variable, it is also called collective in dynamical systems theory. The dynamics of phase synchronization are then described by the trajectory $\Phi_{n, m}(t)$ and its attractors determine the collective behavior of the coupled dynamical system.

\subsubsection{From coupled oscillators to movement coordination}

Following the definition of phase synchronization, the concept of our systematic approach to model HHI is outlined in Fig. 2. Each of the action tasks 1 and 2 is repre- 
sented by the agent's end effector positions over time, which we call the motion trajectory in the following. Since the interaction of the coupled oscillators is described by the evolution of its phases, the transformation between motion trajectories and phases is required. It provides the phase of an action task, which models one agent's observation of the other's action. If the transformation is bijective, it can also provide the motion trajectory of a robotic agent and model the generation of actions in HRI, see e.g. Lorenz et al. [2011] for our experimental setup for investigation of human-robot movement coordination. Within this study, we restrict the investigation of these transformations to model action observation in HHI.

Note: As indicated in Fig. 2, the agents' action tasks do not necessarily need to be identical, as long as the motion trajectories can be transformed to phase representations.

Each agent's individual behavior regarding the task progress is represented by a self-sustained phase oscillator with a constant natural frequency. Interaction is modeled through the coupling function, hence temporal coordination patterns as a result of the agents' joint behavior are resembled by phase synchronized oscillators. Both the above transformations as the required analytical tool and the dynamical system model designed to capture the agents' interactive behavior are presented in the following.

\section{Analytical methods}

In this section, we provide a set of analytical tools to capture and characterize movement synchronization between the agents, a prerequisite to the model design. Note that for reasons of clarity, the presented methods are derived for a single agent unless otherwise stated. If necessary, subscripts 1,2 are used to indicate correspondence to agent 1 and 2 respectively. For illustration purposes, parts of the presented methods are exemplified based on the experimental data.

\subsection{Data reduction to the effective task space}

The experimental data gathered within this study consist of the agents' end effector positions over time, i.e. the motion trajectories expressed in a three-dimensional Cartesian frame $C$ (see Fig. 3). Data complexity can be reduced to simplify the analysis, if only the information of the data required for a minimal description of the task is kept. The three-dimensional motion trajectory of each agent is projected into a one-dimensional subspace spanned by the vector difference of the respective target and starting point, as illustrated in Fig. 3. This projection preserves the temporal relation of the agents' end effectors regarding the action task, and thus represents the effective task space. Further analysis is conducted based on the one-dimensional motion trajectory $x(t)$. The move-

ment onset is defined as the instant of time when the difference between the position of the end effector $x$ and its initial starting position exceeds $5 \mathrm{~mm}$ for the first time.

\subsection{Transforming the motion trajectory to phase}

In order to investigate dyadic phase synchronization, we present three methods transforming the motion trajectory $x(t)$ into a phase $\theta(t)$. Since we are not only interested 


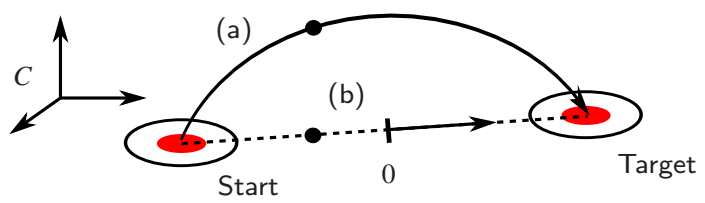

Figure 3: (a) Sample three-dimensional motion trajectory and (b) projected onedimensional trajectory $\mathrm{x}(\mathrm{t})$ between the agent's start and target point. The origin 0 of the task space is set such that $x(t)$ is zero-mean

in steady-state synchronization, but also in the potentially transient process leading to phase synchronization, appropriate techniques have to be able to reflect non-stationary features of the data into an instantaneous phase.

\subsubsection{State-space method}

Assuming harmonic or quasi-harmonic movements, a two-dimensional state-space $(x, \dot{x})$ can be defined. It is sufficient to describe the state of a one-dimensional oscillatory system. When the motion trajectory is plotted in its state space, the cyclic nature of the movements becomes obvious, see Fig. 4. The phase of the oscillatory system

$$
\theta(t)=\arctan \left(\frac{{ }^{n} \dot{x}(t)}{-{ }^{n} x(t)}\right),
$$

can be extracted from the state-space trajectory, with

$$
{ }^{n} \dot{x}(t)=\frac{\dot{x}(t)}{|\hat{\dot{x}}|} \text { and }^{n} x(t)=\frac{x(t)}{|\hat{x}|}
$$

being the normalized velocity and position. The constants $\hat{\dot{x}}$ and $\hat{x}$ denote the extrema of the velocity and position respectively observed in the motion trajectory, which can be updated on-line after each half-cycle. In this context, Varlet and Richardson [2011] provide a benchmark on continuous phase computation using non-stationary, oscillatory test signals and emphasize the superior performance when half-cycle normalization is applied. The state normalization provides a phase characteristics widely independent of the actual physical constraints of human movements, i.e. the peak amplitudes and velocities.

Note: The state-space method is especially well-suited for real-time analysis as long as the motion trajectory is quasi-harmonic. For more complex tasks, the statespace trajectories will differ from simple circular shapes. In such case, the only way to obtain a meaningful phase with this method is band-pass filtering, which selects only a single frequency component or feature of the motion trajectory respectively to be represented in the phase. If the measurements are noisy, low-pass filtering of $\dot{x}$ may be required, which introduces an additional phase lag in real-time estimation. 


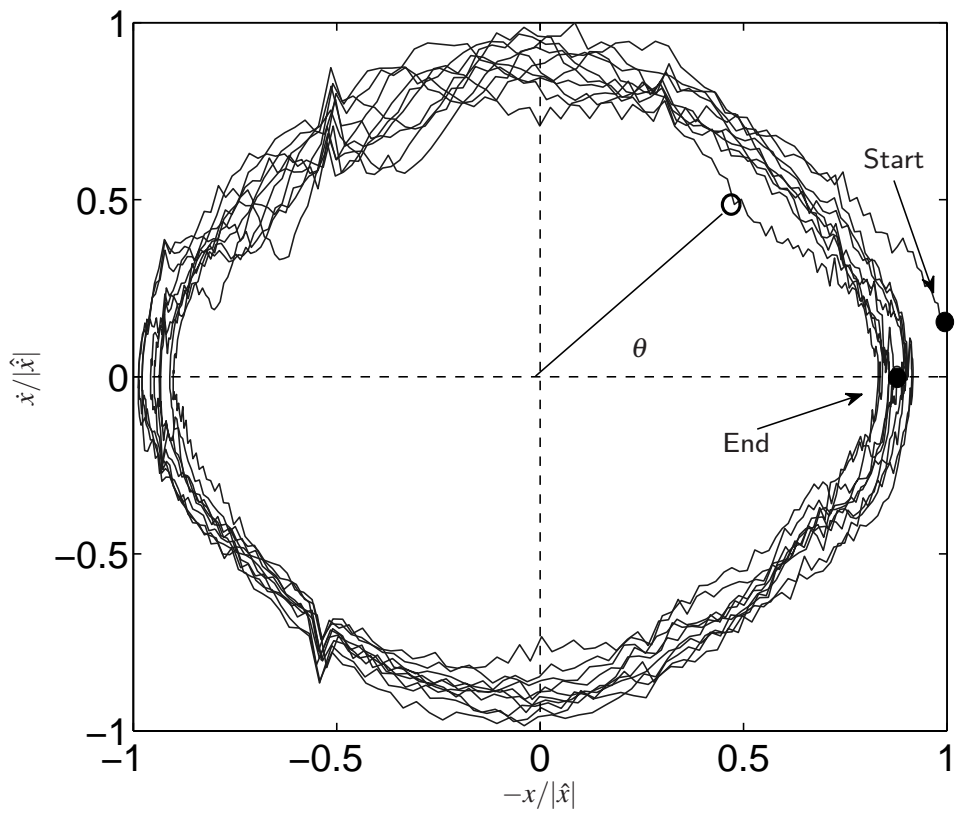

Figure 4: Normalized state-space trajectory for an experimental trial with ten cycles, where the normalized velocity is plotted against the normalized position. Trajectories form a closed curve with an approximately circular shape between movement start and end. The position $x$ is inverted to obtain a phase $\theta$ increasing over time

\subsubsection{Spectral method}

Comparisons of the spectral signal analysis methods (short-time) Fourier-, Hilbert- and wavelet-transform show equivalent results due to their formal equivalence when their filter kernels are parameterized respectively [Bruns, 2004]. In this article, we adopt the analytic signal concept based on the Hilbert transform for computing a phase [Rosenblum and Kurths, 2007]. The Hilbert transform provides the instantaneous phase and amplitude of the signal ${ }^{1} x(t)$ via construction of an analytic signal $\zeta(t)$, which is a complex function of time defined as

$$
\zeta(t)=x(t)+j \tilde{x}(t)=A(t) e^{j \theta(t)},
$$

where $\tilde{x}(t)$ is the Hilbert transform of $x(t)$. It is given by

$$
\tilde{x}(t)=\frac{1}{\pi} \mathrm{P} . \mathrm{V} \cdot \int_{-\infty}^{\infty} \frac{x(\tau)}{t-\tau} d \tau
$$

where P.V. means that the integral is taken in the sense of the Cauchy principle value. The instantaneous amplitude $A(t)$ and phase $\theta(t)$ of the signal $x(t)$ are uniquely defined

\footnotetext{
${ }^{1}$ The motion trajectory $x(t)$ is treated as a signal.
} 
by (5). Since we focus on the analysis of phase synchronization, we are interested in the phase only given by

$$
\theta(t)=\arg (\zeta(t)) .
$$

Fourier-spectra of the motion trajectories $x$ captured in our experiment show a single sharp peak denoting the mean cycle frequency. Thus, the motion trajectories can be treated as narrow band signals and a meaningful phase can be obtained via the spectral method.

Note: The spectral method is nonlocal in time due to the infinite integral bounds in (6). Therefore, its applicability to real-time phase estimation is limited, although it is a well-suited tool for off-line analysis. Both the state-space and the spectral method presented above require zero-mean motion trajectories to obtain phases that cover an angular range of $\pi$ per half-cycle, i.e. the movement from the starting to the target point or vice versa respectively.

\subsubsection{Hybrid method}

Both the state-space and spectral method perform well only for quasi-harmonic motion trajectories. In our experimental task paradigm, this requirement is fulfilled. However, repetitive action tasks generally comprise a sequence of heterogeneous action primitives which compose the overall motion trajectory, such as elementary point-topoint movements, or even static dwelling periods where the position remains rather constant. Inspired by the fundamental signal-theoretic idea to capture an oscillation's cyclic progress in a continuous instantaneous phase, a novel approach is proposed here that extends the notion of phase to describe the instantaneous progress of an arbitrary cyclic action. The goal is to construct an instantaneous, linear phase $\theta(t)$ in $[0,2 \pi]$ from the motion trajectory $x(t)$ previously observed for a single cycle in time $t=[0, T]$ with cycle period $T$. First, the trajectory has to be segmented into a sequence of $P$ action primitives such that for each primitive $p=1 \ldots P$ an invertible representation

$$
x_{p}(t)=f_{p}\left(\chi_{p}\right)
$$

can be found, where $x_{p}(t)$ approximates the motion trajectory and $\chi_{p}$ represents the relative time in $p$. In a second step, a piecewise continuous phase

$$
\theta_{p}(t)=K_{p} f_{p}^{-1}\left(x_{p}(t)\right)
$$

can be obtained, where $f_{p}^{-1}$ is the inverse of $f_{p}$ and $K_{p}$ is a factor scaling $\chi_{p}$ to angular values. Guided by switching conditions between the primitives which can be derived from the segmentation, the phase $\theta_{p}(t)$ of the current primitive is determined by (9) and accumulated over $p$ in order to obtain a continuous phase $\theta(t)$ of the whole motion trajectory.

In the following, we exemplify the idea of the hybrid method. In Fig. 5, a close-up of a representative trajectory showing the first cycle is given. To assure that invertible representations (8) exist, the motion trajectory can be segmented into four primitives per cycle and parameterized as follows: the forward movement $(p=1)$ in time $T_{f}(k)$, the dwell at the target point $(p=2)$ for $T_{t}(k)$, the backward movement $(p=3)$ in $T_{b}(k)$, 


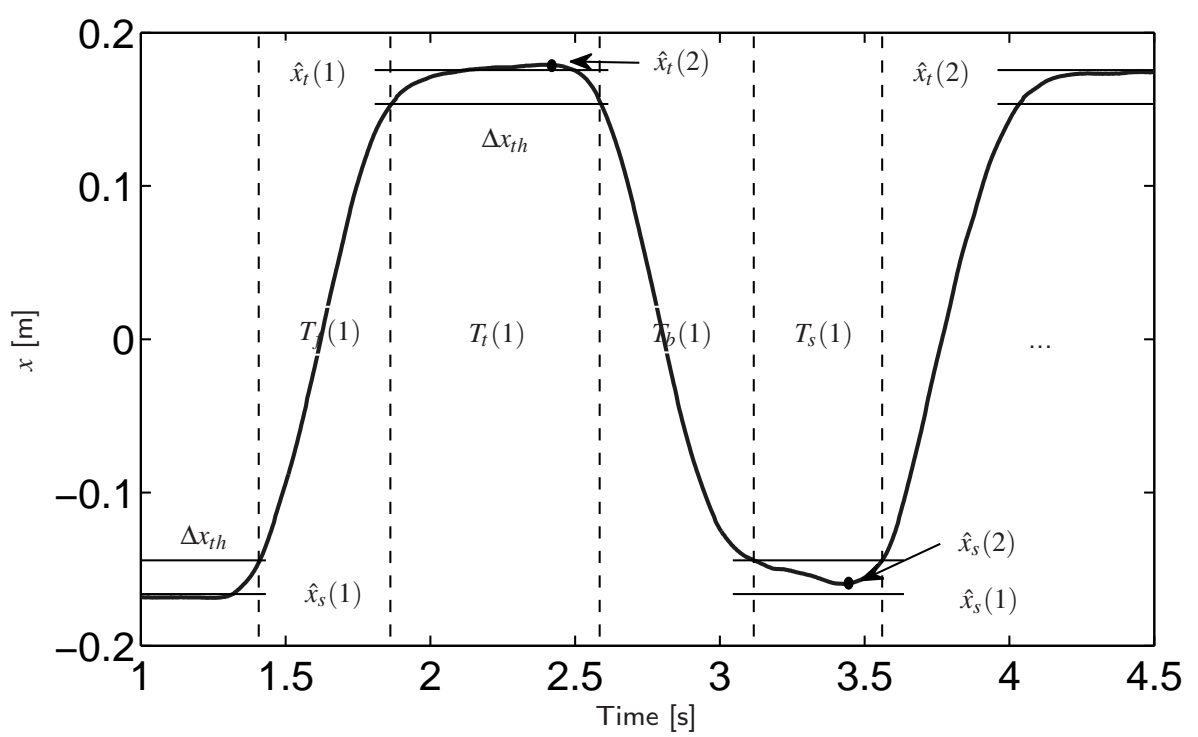

Figure 5: The first cycle of a representative trajectory with the durations and parameters used for segmentation. Vertical dashed lines indicate the threshold-based segmentation into four primitives per cycle. The dwelling periods are considered as separate action primitives

and the dwell at the starting point $(p=4)$ for $T_{s}(k)$, where $k$ denotes the cycle index. This kind of trajectory segmentation can be performed on-line based on a threshold $\Delta x_{t h}$ with respect to the start and target extrema $\hat{x}_{s}(k)$ and $\hat{x}_{t}(k)$, as visualized by horizontal lines in Fig. 5.

A possible representation (8) for primitive 1 is the minimum jerk model validated for point-to-point hand movements by Flash and Hogan [1985]. It approximates the motion trajectory

$$
x_{1}(t)=\left(\hat{x}_{t}(k)-\hat{x}_{s}(k)\right) g\left(\chi_{1}\right)+\hat{x}_{s}(k),
$$

with the fifth-order polynomial

$$
g\left(\chi_{1}\right)=6 \chi_{1}^{5}-15 \chi_{1}^{4}+10 \chi_{1}^{3}
$$

minimizing jerk, and $\chi_{1} \in[0,1]$ being the relative movement time. In a next step, the minimum jerk model needs to be inverted, which can be done by finding the real root $\chi_{1}$ of the polynomial (11) in each time step. The phase for primitive 1 can then be written as

$$
\theta_{1}(t)=K_{1} \frac{\chi_{1}-\chi_{t h}}{1-2 \chi_{t h}}
$$

with the scaling factor

$$
K_{1}=\frac{2 \pi T_{f}(1)}{T(1)}
$$


depending on the movement time $T_{f}(1)$ and the cycle period $T(1)$. Note that the threshold-based segmentation cuts off the beginning and the end of motion, thus in (12) the relative time $\chi_{1}$ is re-normalized by a transformation with $\chi_{t h}$. The value $\chi_{t h}$ is the real root of (11) at the value $\Delta x_{t h}$.

Since the primitive 2 is characterized by dwelling without considerable motion, a phase representation purely depending on time is proposed

$$
\theta_{2}(t)=K_{2} \chi_{2}+\theta_{1},
$$

with the scaling factor

$$
K_{2}=\frac{2 \pi-\theta_{1}}{T(1)-T_{f}(1)} .
$$

Note that here, $\chi_{2}$ is the relative time w.r.t. the primitive entry. The offset $\theta_{1}$ in (14) accounts for the phase accumulated previously in primitive 1 and enables a continuous switching of the primitives. Due to symmetry of the primitive sequence, the phases $\theta_{3}(t)$ and $\theta_{4}(t)$ are calculated analogously to $\theta_{1}(t)$ and $\theta_{2}(t)$. If the primitive durations vary between cycles, the phase does not exactly evaluate $2 \pi k$ after the completion of cycle $k$.

Note: Arbitrary complex action sequences can be described on-line with the hybrid method if a feasible segmentation and hybrid representation of the action primitives is found. The estimated phase is an indicator of a repetitive task's relative temporal progress, which is affected however by the chosen parameterization. The approach can be enhanced by learning and prediction techniques to improve the estimation performance.

\subsection{Measuring synchronization}

In the following, the analytical tools and requirements to detect and measure synchronization based on the agents' phases $\theta_{1}(t)$ and $\theta_{2}(t)$ are presented.

\subsubsection{Relating the phases - dyadic phase difference}

Synchronization between the agents can be detected when their phases are related to each other through the generalized phase difference $\Phi_{n, m}(t)$ given in the definition of synchronization (3). In general, boundedness of the phase difference can be found also for different cycle frequencies of the coupled oscillatory system. For example, one agent performing one cycle while the other completing two cycles can be still referred to synchronization, which would be called synchronization of order 1:2. The participants in our experiment were performing at similar cycle frequencies, which is indicated by the standard deviation $\mathrm{SD}=0.12 \mathrm{~Hz}$ at a mean $\mathrm{M}=0.73 \mathrm{~Hz}$ over all trials. Thus, the analysis of synchronization can be restricted to the order $1: 1$ by calculating the dyadic phase difference

$$
\Phi(t)=\Phi_{1,1}(t)=\theta_{1}(t)-\theta_{2}(t) .
$$

Note: Since the phase representations in Sec. 3.2 yield angular values defined on the circle $[0,2 \pi]$, the phase is unwrapped, i.e. $2 \pi$-jumps are removed such that $\Phi \in \mathbb{R}$. 
The time series $\Phi(t)$ start at the movement onset of the delayed agent and end after completion of ten cycles.

\subsubsection{Experimental observation of synchronization}

In theory, a coupled oscillatory system is synchronized, if its phase difference stays bounded for all times. However in our experimental setting, we are obliged to apply a weaker criterion to investigate synchronization due to a limited available observation length of human movements: a thorough trade-off between capturing a potential process of phase convergence and the influence of the participants' fatigue increasing over time has to be made. With an observation length of ten cycles chosen in our experiment, we are able to show that the transient process of synchronization is happening in the first few cycles of the task. The temporal differences between the actions of both agents at key events, such as the time of target entry are decreasing within the first three cycles only [see also Lorenz et al., 2011], which makes a length of ten cycles a reasonable choice.

\subsubsection{Quantification with the synchronization index}

Numerous approaches on measuring synchronization from time series can be found in literature, see e.g. Kreuz et al. [2007] for a comprehensive comparison. Since the instantaneous phases represent the oscillatory entities in this article, we follow a common approach to quantify phase synchronization: given the time series $\Phi(t)$ consisting of $N$ directional observations $\Phi\left(t_{j}\right)$, directional statistics provides a synchronization index

$$
S I=\left|\frac{1}{N} \sum_{j=1}^{N} e^{i \Phi\left(t_{j}\right)}\right|=1-\mathrm{CV}
$$

where CV denotes the circular variance of an angular distribution. ${ }^{2}$ The synchronization index $S I$ is the length of the mean resultant vector of the phase difference samples $\Phi\left(t_{j}\right)$ transformed into unit vectors in the complex plane.

Note: $S I$ lies in the interval [0,1]. Given a perfectly uniform distribution of phase differences, it would equal zero. In perfect synchronization it would equal one, which means that all samples of $\Phi$ point to the same direction.

\section{Coordination model of the interacting dyad}

In this section, we present the steps taken towards a model of interpersonal movement coordination. Starting with an investigation of the experimental data regarding the characteristics of movement synchronization, the model structure is developed and the model parameters are identified.

\footnotetext{
${ }^{2}$ the synchronization index $S I$ is also called mean phase coherence.
} 


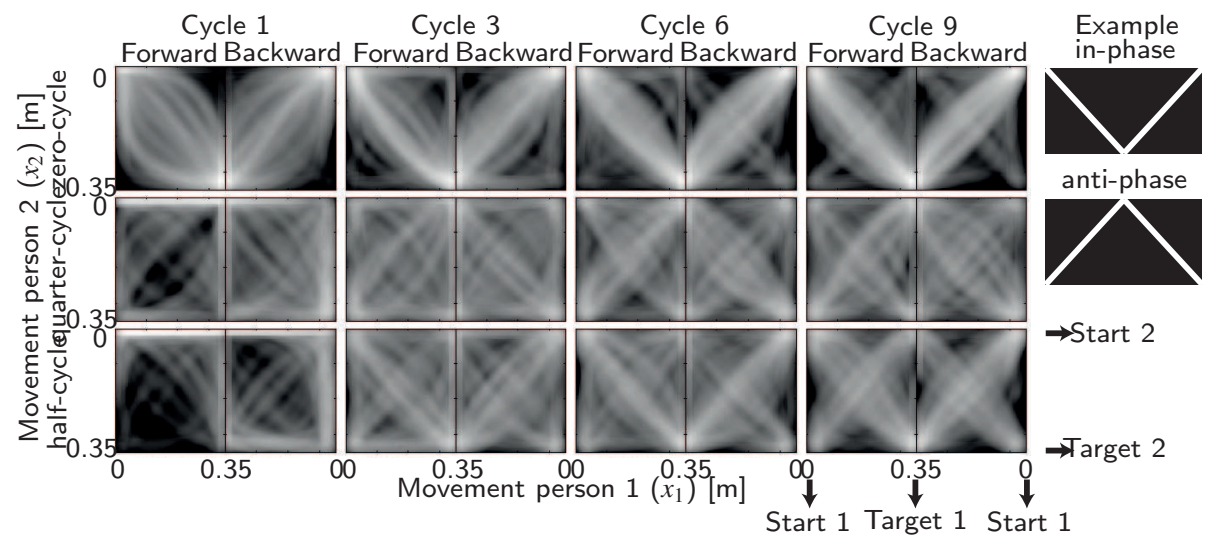

Figure 6: How did synchrony evolve? This Figure contains a frequency distributions (of all trials) of the hand position of person 2 relative to that of person 1 [see also Lorenz et al., 2011]. The hand positions are one-dimensional positions between the start $(=0 \mathrm{~m})$ and target $(=0.35 \mathrm{~m})$. The brighter a location in this picture is, the more often that positional relationship occurred. Relative movements (between the two participants) show up as lines. Perfect in-phase and anti-phase synchrony would be straight diagonal lines, see upper right. The pictures show that the participants tended to synchronize already early in the trial. Data are plotted separately for the first, third, sixth and ninth cycle. In the zero condition (upper panels), phase relationships already appear like in-phase and anti-phase relationships in the third cycle. In the other two conditions (quarter-cycle in the middle panels and half cycle conditions in the lower panel) roughly the same happens, but there appear to be slightly stronger and more deviations

\subsection{Experimental results}

In a first step, we elucidate the temporal process leading to synchronization and the phase relations emerging among the partners by plotting the data and analyzing the phase difference.

\subsubsection{Patterns of movement synchronization}

In our study of human movement behavior in a goal-directed task we found relations between the movements of dyads which were established over the course of a trial [Lorenz et al., 2011]. In order to visualize these findings, a frequency distribution is plotted as a heat map of all movement signals. Here the movement $x_{2}$ of person 2 is represented as a function of the movement $x_{1}$ of person 1. For each trial and cycle the actual position of person 2 is calculated as a function of person 1's position. The resulting curve is sampled with an underlying grid of $100 \times 100$ cells and the number of times each cell in the grid is hit by a curve is determined. Those binned counts are plotted for each forward and backwards movement separately into a heat map in which brightness codes frequency of cell hits, see Fig. 6 . In these plots, perfect 
in-phase or anti-phase synchrony appear as straight diagonal lines. During in-phase relation the movements have no phase shift, e.g. if person 1 is in the starting position at $0 \mathrm{~m}$ person 2 is there as well. On their way to the target participants are always at the same point in their trajectory. Thus, when dyads are perfectly in-phase during forward movements, a line goes from top left to bottom right. During backwards movementswhere the abscissa labeling goes down-the plot shows a straight line from the bottom left to the top right if the interaction partners are perfectly synchronized. In perfect anti-phase, this pattern is mirrored horizontally because when one person is at the start position, the other person is in the target position and vice versa. When participants are not perfectly synchronized, data appear curved. Note that only the in-phase and the anti-phase relation are easy to identify in this graphical representation because they appear as straight lines. Other stable phase relations would show a more complicated pattern and thus can not be determined as intuitively.

Most importantly, the heat map visualizes that in-phase and anti-phase relationships are already established early in the trial. During the first cycle, data tend to be curved for all conditions. After that, movements are quickly adapted, which is depicted by the curves becoming straighter. Data of the zero-cycle condition shows that people are already almost perfectly adapted to each other after performing the third cycle. Straight lines become prevalent indicating in-phase movements. In contrast, during the half-cycle condition both the in-phase and anti-phase relation are established roughly equally often until the ninth cycle. This is interesting because one might have expected that if starting off at the same time mainly leads to in-phase relations, starting off at opposite positions should mainly lead to anti-phase relations. Roughly the same result is observed for the quarter-cycle condition. The difference to half-cycle is that in comparison more curved lines appear during the quarter-cycle condition and constant phase relations are established later there. This indicates that it is more difficult to establish a stable in-phase or anti-phase relation when starting off in an odd temporal ratio.

\subsubsection{Characterization of the attractor regimes}

In addition to the graphical representation of synchronization in the heat maps, the analysis of the phase difference allows to further characterize the attractive domains of the synchronization process.

Any prevalent phase relation can be made visible by histogram representations of the phase difference time series, see Fig. 7 (a)-(c). Since the attractor regime is characterized by the relative phase relation within the dyad, we define the relative phase difference

$$
\Phi_{r}(t)= \begin{cases}|\Phi(t) \bmod 2 \pi|, & \text { if }|\Phi(t) \bmod 2 \pi| \leq \pi \\ 2 \pi-|\Phi(t) \bmod 2 \pi|, & \text { otherwise. }\end{cases}
$$

The data samples of $\Phi_{r}(t)$ are assigned to 40 equally spaced bins in $[0, \pi]$ and accumulated over all trials. Distinct peaks at angular values around multiples of $\pi$ are common to the histograms of the three conditions zero-cycle, quarter-cycle and half-cycle. They become even sharper if only the second half of each trial is considered where 


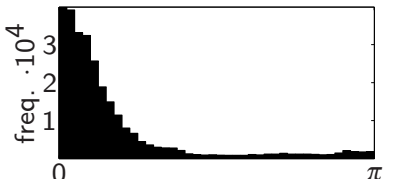

(a) zero-cycle $\Phi_{r}[\mathrm{rad}]$

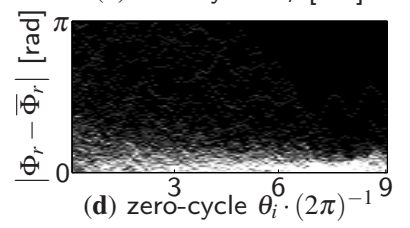

Figure 7: The histograms (a)-(c) of the relative phase difference $\Phi_{r}$ show clear peaks at values around 0 and $\pi$, which suggest multiples of $\pi$ as preferred phase relations or attractors respectively. The heat maps (d)-(f) depict the transient process of the relative phase difference towards its attractor (ordinate), which is indexed by the phase angle of the delayed person $\theta_{i}$ (abscissa). Frequency of the relative phase difference is coded by brightness. The time series of $\Phi_{r}$ and $\theta_{i}$ are generated with the spectral method and accumulated over all trials in the three conditions

transient processes are nearly completed. Totally uncorrelated phases would cause approximately uniform distributions, whereas perfectly synchronized phases would result in sharp vertical lines. The center values of such peaks can thus be treated as features appropriate for modeling. The width of the peaks is associated with the variance and the synchronization index (17) respectively.

With a closer look at the distributions, it can be stated that in the zero-cycle condition (Fig. 7a) the participants' phase difference predominantly stays in one single regime at $\Phi_{r}=0$, which refers to the in-phase relation. Starting off in-phase means attaining an in-phase relation in nearly all of the trials. In the quarter-cycle condition (Fig. 7b), two different attractor regimes become visible: the in-phase attractor but also an attractor at $\Phi_{r}=\pi$, which refers to the anti-phase relation. Starting off in quarter-cycle leads to both the in-phase and anti-phase relation, while it seems as if the latter was preferred. In condition half-cycle (Fig. 7c), the same attractors as in quartercycle appear. Starting off in half-cycle leads idem to both the in-phase and anti-phase relation.

Note that the histograms only show the prevalent relative phase relations over trials, which are $\Phi_{r}=0$ and $\Phi_{r}=\pi$. Though two attractors in Fig. 7(b) and (c) can be clearly identified, it can not be determined from the histograms if spontaneous switches between them occur within trials. Therefore, we investigate the convergence of the relative phase difference $\left|\Phi_{r}(t)-\overline{\Phi_{r}}\right|$ as a function of cycles, where

$$
\overline{\Phi_{r}}=\frac{1}{N-\left\lfloor\frac{2 N}{3}\right\rfloor+1} \sum_{j=\left\lfloor\frac{2 N}{3}\right\rfloor}^{N} \Phi_{r}\left(t_{j}\right)
$$

denotes the mean of each time series $\Phi_{r}(t)$ taken over the last third of the total samples $N$ of each trial. The distributions in Fig. 7(d)-(f) are nicely aligned around zero 


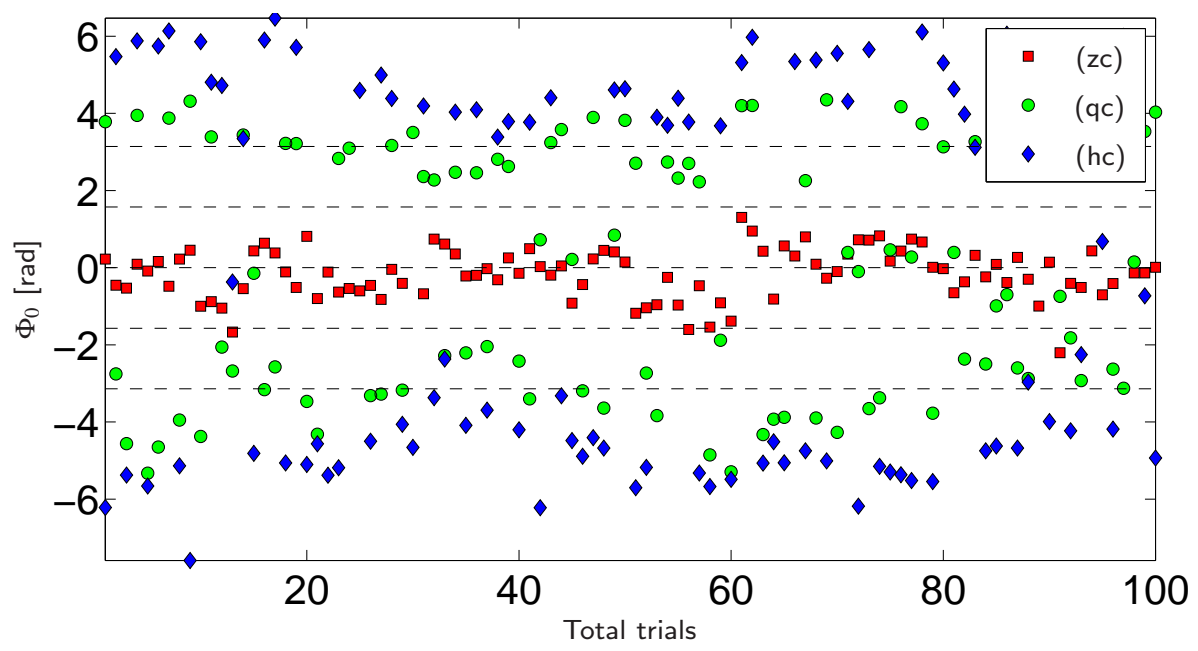

Figure 8: Actual initial phase differences $\Phi_{0}=\Phi(t=0)$ over trials calculated with the spectral method for the conditions zero-cycle (zc), quarter-cycle (qc) and halfcycle (hc). Time $t=0$ dentoes the movement onset of the delayed person. Dashed lines indicate the phase relations triggered by the start off conditions

between cycle 6 and 9 for most of the trials, which illustrates overall convergence to the preferred attractors. The distributions are scattered between 0 and $\pi$ up to cycle 6 , as an indication of the process of convergence happening in the first few cycles of interaction. No preferred clusters around $\pi$ can be detected in these distributions. Thus, we can conclude that besides the initial convergent process, within-trial switches between the two preferred attractors rarely take place during the experimental observation length.

Both the heat map representation and the phase difference distribution provide clear evidence of synchronization in our goal-directed experimental task. The process of synchronization is characterized by two attractor points of the relative phase difference, namely the in-phase and the anti-phase relation.

\subsubsection{Initial phase difference}

Though in the half-cycle condition the dyads are triggered to start of in the anti-phase relation, both the anti-phase and the in-phase attractor can be identified in the histogram 7(c), with even a prevalence of the latter. For clarification, the actual initial phase difference of each trial is illustrated in Fig. 8. The distribution shows clusters for the different conditions, yet under quarter-cycle and half-cycle with an additional phase delay around $\pi$ with respect to the phase relation triggered by the respective start off condition. These delays are mainly caused by the participants' reaction and dwell times when engaging jointly in action. Especially in the half-cycle condition, the actual initial phase differences are often close to the in-phase relation, which explains the frequently appearing in-phase attractor in the histogram 7(c). Due to the simultaneous 


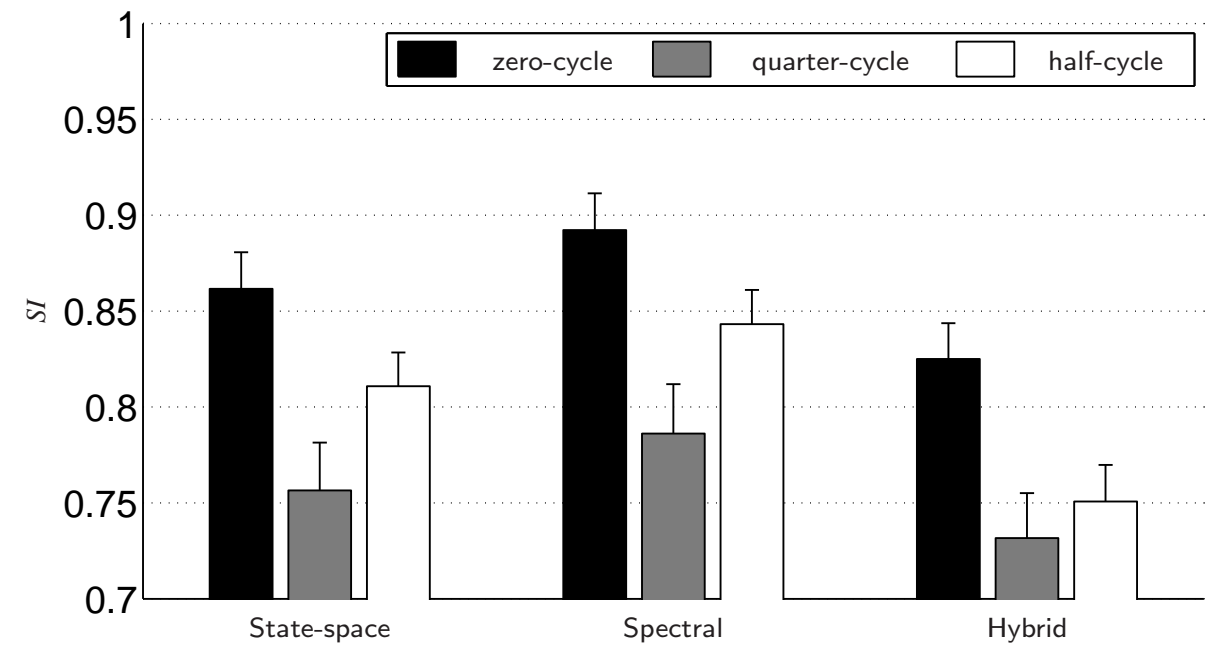

Figure 9: Synchronization index SI for the conditions zero-cycle, quarter-cycle and half-cycle averaged over all trials. For comparison, SI is calculated separately for the phases estimated by the state-space, spectral and hybrid method. The bars represent standard errors of the means

start off trigger in the zero-cycle condition, the actual initial phase difference is affected less by the delay. In brief, the applied conditions are shown to effectively trigger a distribution of different initial phase relations, which enables to perform a parameter estimation based on the response dynamics of the dyads.

Note: In goal-directed tasks, a leadership among the dyad can be defined when the sign of the phase difference $\Phi=\theta_{1}-\theta_{2}$ is evaluated: person 1's phase $\theta_{1}$ larger than person 2's phase $\theta_{2}$ means that person 1 is leading the task by preceding person 2's action and vice versa. The symmetry of $\Phi_{0}$ observed in Fig. 8 is due to the initial trigger of action start off, which is equally often assigned to each of the partners.

\subsubsection{Evaluation of the synchronization index}

The synchronization index SI given by (17) provides a quantification of synchronization in a single number. Based on the definition that a collective remaining constant over time yields the highest degree of synchronization, the measure penalizes any variability of the collective including e.g. the transient process when getting synchronized and also transitions between attractors. Therefore, it has to be considered that the choice of the variables constituting the system's collective affects the characteristics of the measure. When looking at the scores of the synchronization index SI in Fig. 9, the influence of the method used to estimate the phase variables becomes obvious. A oneway repeated measures ANOVA was performed to assess the difference between SIs obtained with the state-space, spectral and hybrid method. As the sphericity criterion was not met, Greenhouse-Geisser correction was applied. SIs differed quantitatively, $F(1.2,34.9)=66.78, p<.001$, which can be explained by a differing sensitivity of the 
method to the variabilities in the movement profiles. Only the dominating frequency determines the phase of the spectral method $(M=0.84)$, which makes it rather insensitive to small, local movement variabilities. In contrast, such variabilities directly influence the instantaneous phase of both the state-space $(M=0.81)$ and the hybrid method $(M=0.77)$. The inferior score of the hybrid method suggests that the parameterization of the minimum jerk model chosen as the primitive representation does not perfectly match the movement profiles observed in our experiment.

The indexes given in Fig. 9 consider whole trials including the transient process of synchronization. If the transient process is neglected and the synchronization index is calculated only on the second half of each trial, similar qualitative results can be obtained. In condition zero-cycle, the highest index values are achieved on average. Thus, starting off in zero-cycle enables the partners to reach highest quality of synchronization. Starting off in quarter-cycle yields lowest values due to a rather high phase variability within the dyads, which is in agreement with the distribution Fig. 7(b). Medium index values are attained in half-cycle. The resulting indexes show similar trends within methods, yet the one-way repeated measures ANOVAs with the within-subject factor condition (zero-cycle, quarter-cycle, half-cycle) performed for every method did not reveal any significant differences between start off conditions, all $p>.05$. If we interpret the synchronization index as an analytical indication of strength of the involved attractors, it can not be clearly stated that the attractor strengths of the in-phase and the anti-phase relation differ in our goal-directed experiment. In contrast, research work on interpersonal movement synchronization in purely rhythmic tasks provides evidence of different attractor strengths, i.e. the anti-phase attractor has a strong tendency to be weaker than the in-phase attractor or even disappears [Schmidt and O'Brien, 1997, Richardson et al., 2007].

\subsection{Modeling approach}

The analysis of the phase variables reveals temporal interaction between the partners which is closely related to the definition of phase synchronization. The emerging synchronization effects can be modeled as a synchronization problem of two mutually coupled oscillators. In a next step towards the coordination model, we set up an adequate model structure and investigate its relevant properties.

\subsubsection{Model of two coupled oscillators}

First we recall the general equations of motion of two limit cycle oscillators (1) and (2) that are mutually coupled. Under the assumption that the coupling functions $G_{i}$ are weak and the oscillatory dynamics are harmonic [Kuramoto, 1984], the above equations can be reduced to a simpler set written in terms of the oscillators' phase angles $\theta_{i}$ as

$$
\begin{aligned}
& \dot{\theta}_{1}=\omega_{1}+H_{1}\left(\theta_{2}-\theta_{1}\right) \\
& \dot{\theta}_{2}=\omega_{2}+H_{2}\left(\theta_{1}-\theta_{2}\right),
\end{aligned}
$$

where $\omega_{i}$ are the natural frequencies of the oscillators and the coupling functions $H_{i}$ depend on the phase difference between the oscillators. 
Note: In our modeling approach the phase $\theta_{i}(t)$ is assumed to be quasi-harmonic. Harmonicity is either fulfilled due to the harmonic nature of the movements the phase is constructed from or it can be achieved by an appropriate phase transformation, e.g. the hybrid method presented in Section 3.2.3.

\subsubsection{Extending the Kuramoto model}

Several candidates for the coupling functions $H_{i}$ in (18), (19) have been proposed in the context of movement synchronization. One of them is the sinusoidal function proposed by Rand et al. [1988] which yields the model equations

$$
\begin{aligned}
& \dot{\theta}_{1}=\omega_{1}+K \sin \left(\theta_{2}-\theta_{1}\right) \\
& \dot{\theta}_{2}=\omega_{2}+K \sin \left(\theta_{1}-\theta_{2}\right) .
\end{aligned}
$$

It is also known as the classical Kuramoto model [Kuramoto, 1984], where $K$ is the coupling gain between the oscillators. It is assumed to be isotropic for both oscillators. We adopt the model of coupled Kuramoto oscillators for the following reasons:

- Despite its simplicity, the observed main effects of synchronization are replicated: The natural frequencies refer to the agents' individual frequency levels as an individual behavior, whereas the additive non-linear coupling term allows synchronization between the agents to emerge.

- Emerging synchronization is explained as an effect of co-adaptation with an isotropic bidirectional coupling. Directionality of coupling characteristics would be hard to identify from short-time bivariate data recorded during natural HHI, since unsynchronized regimes have to be observed extensively [Smirnov and Andrzejak, 2005].

- The goal-directedness of the task constrains the amplitudes of movements, thus only the quasi-harmonic phases are considered. The two-degrees-of-freedom model fosters parameter identification from noisy data.

The phase difference dynamics between the two oscillators are obtained by subtracting (21) from (20) and can be compactly written as

$$
\dot{\Phi}=\Delta \omega-2 K \sin \Phi
$$

with $\dot{\Phi}=\dot{\theta}_{1}-\dot{\theta}_{2}$ and the frequency detuning

$$
\Delta \omega=\omega_{1}-\omega_{2}
$$

The analytical results from Sect. 4.1 show that the phase relation between the interacting agents ends up predominantly either in in-phase $(\Phi=0)$ or in anti-phase $(\Phi=\pi)$. Therefore, we extend the phase difference dynamics (22) by two additional equilibrium points per period of $\Phi$, which yields the differential equation of the phase difference

$$
\dot{\Phi}=h(\Phi)=\Delta \omega-2 K \sin (2 \Phi)
$$


with the model equations

$$
\begin{aligned}
& \dot{\theta}_{1}=\omega_{1}+K \sin \left[2\left(\theta_{2}-\theta_{1}\right)\right] \\
& \dot{\theta}_{2}=\omega_{2}+K \sin \left[2\left(\theta_{1}-\theta_{2}\right)\right],
\end{aligned}
$$

called the extended Kuramoto model in the following.

\subsubsection{Stability analysis}

Next, the stability of the equilibrium points of the extended Kuramoto model (24) is investigated. Setting $\dot{\Phi}=0$, one obtains

$$
2 K \sin \left(2 \Phi_{e}\right)=\Delta \omega .
$$

The equilibrium points $\Phi_{e}$ are then given by the solutions of (27) or graphically represented, by the points of intersection of the curves $2 K \sin (2 \Phi)$ and $\Delta \omega$, see Fig. 10.

Note: Equilibrium points $\Phi_{e}$, i.e. solutions of (27) exist, if the parameter set $(\Delta \omega, K)$ satisfies the inequality

$$
|\Delta \omega|-2|K|<0 .
$$

Let $\eta=\Phi-\Phi_{e}$ be a small perturbation away from $\Phi_{e}$. In order to analyze the stability of the equilibrium points, we investigate whether the perturbation grows or decays. First we derive a differential equation for $\eta$ given by

$$
\dot{\eta}=\frac{d}{d t}\left(\Phi-\Phi_{e}\right)=\dot{\Phi}=h\left(\Phi_{e}+\eta\right)
$$

Using Taylor's expansion and noting that $h\left(\Phi_{e}\right)=0$ one gets

$$
\dot{\eta}=\eta h^{\prime}\left(\Phi_{e}\right)+O\left(\eta^{2}\right)
$$

where $O\left(\eta^{2}\right)$ gathers quadratically small terms in $\eta$. The gradient

$$
h^{\prime}\left(\Phi_{e}\right)=\left.\frac{d}{d \Phi} h\right|_{\Phi=\Phi_{e}}=-4 K \cos \left(2 \Phi_{e}\right)
$$

denotes the attractor strength of $\Phi_{e}$. If $h^{\prime}\left(\Phi_{e}\right) \neq 0$, the approximation can be written as

$$
\dot{\eta}=\eta h^{\prime}\left(\Phi_{e}\right) \text {. }
$$

It can be seen from (30) that the perturbation $\eta$ grows exponentially, i.e. the equilibrium $\Phi_{e}$ is unstable if $h^{\prime}\left(\Phi_{e}\right)>0$ and decays, i.e. $\Phi_{e}$ is stable if $h^{\prime}\left(\Phi_{e}\right)<0$. The stability analysis can be visualized by investigating the dynamics of the vector field on the abscissa as shown in Fig. 10, where the solid and open dots represent the stable and unstable equilibrium points respectively. Two stable and two unstable equilibrium points exist per period of $\Phi$. The attractor strength $h^{\prime}\left(\Phi_{e}\right)$ given by (29) depends not only on the coupling gain $K$ but also on the frequency detuning $\Delta \omega$, since it shifts $\Phi_{e}$. Growing/decaying values of $\Delta \omega$ move the stable equilibrium points in the positive/negative direction along the abscissa; i.e. the model explains a positive/negative shift of the 


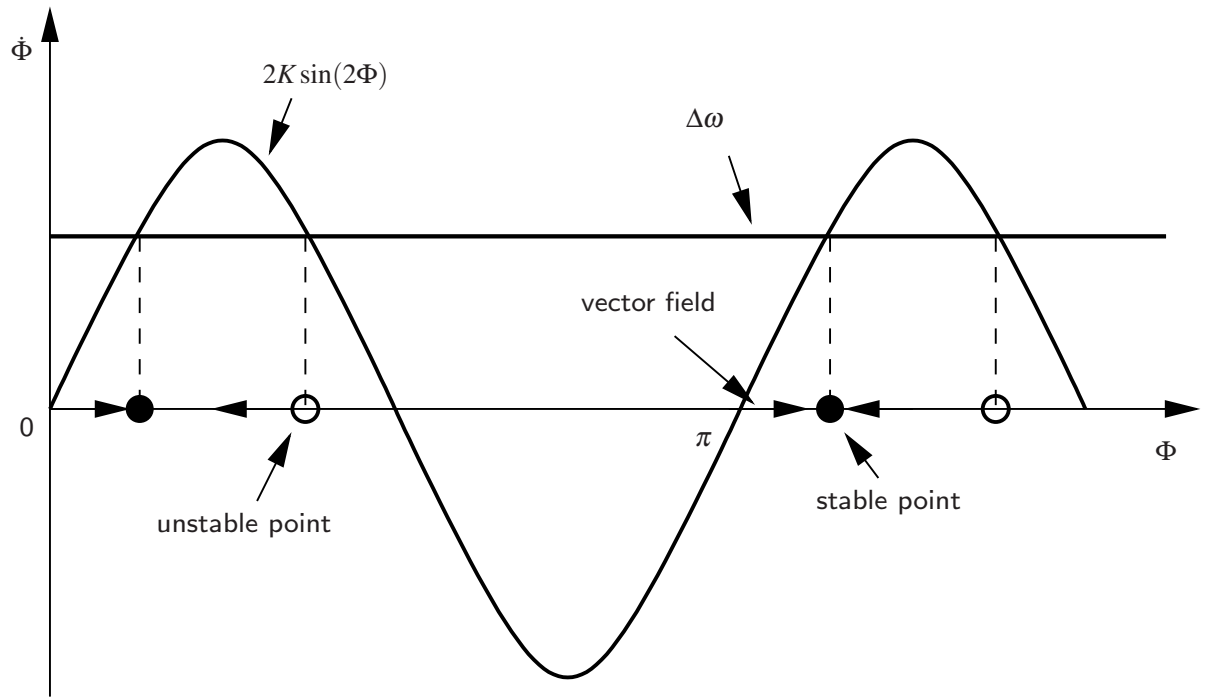

Figure 10: Stability of the equilibrium points of the extended Kuramoto model. The phase difference between the two oscillators converges close to 0 (in-phase) or $\pi$ (anti-phase) depending on the strength of coupling $K$ and the frequency detuning $\Delta \omega$

steady-state phase difference by a positive/negative frequency detuning among the dyad.

Note: The stable equilibrium points around $\Phi=0, \pi$ have the same attractor strength and therefore equal stability properties due to the $2 \pi$-periodicity of (29). The model structure is chosen, since the analysis of the data gathered in our experimental paradigm does not provide clear evidence for attractor switches or different attractor strengths in our goal-directed task. The extended Kuramoto model can be modified to address different attractor strengths, yielding the phase difference dynamics

$$
\dot{\Phi}=\Delta \omega-2 K \sin (2 \Phi)-2 K_{0} \sin (\Phi)
$$

with an additional sine term and coupling parameter $K_{0}$, which is obviously the HKB model structure extended for different eigenfrequencies [Haken et al., 1985, Fuchs and Kelso, 1994].

\subsubsection{Natural frequencies of the oscillators}

Investigation of the relation between the frequency detuning $\Delta \omega$ and the equilibrium frequency $\omega_{e}$ of the coupled oscillators in the extended Kuramoto model allows us to derive the natural frequencies $\omega_{i}$, which describe the uncoupled oscillators. In equilibrium, we can write

$$
\dot{\Phi}=\dot{\theta}_{1}-\dot{\theta}_{2}=0
$$


which is satisfied for the frequency

$$
\omega_{e}=\dot{\theta}_{1}=\dot{\theta}_{2} .
$$

The frequency $\omega_{e}$ is the common frequency during synchronized regimes and can be determined through measurement. Plugging (31) into the model equations (25), (26) and applying (23) yields

$$
\omega_{1,2}=\omega_{e} \pm \frac{1}{2} \Delta \omega .
$$

Thus, $\omega_{e}$ is the mean of $\omega_{1}$ and $\omega_{2}$, which is a common property of the Kuramoto model. The natural frequency $\omega_{i}$ predict agent $i$ 's individually preferred cycle frequency, which is assumed to be a constant parameter within the joint action context. When modeling human motor behavior, the individual cycle frequencies predicted by the model have to be interpreted carefully; it is known for example that working speeds differ when humans perform a motor task alone and jointly with others [see e.g. Vesper et al., 2009].

\subsection{Parameter identification}

In the following, the requirements and technique to obtain the parameter values $(\Delta \omega, K)$ of the model structure and the results of the parameter identification are presented.

\subsubsection{Observation of the natural response dynamics}

A general requirement for a successful parameter identification is the perturbation of the system and the observation of the system's response, which can be achieved either by an externally applied excitation or by an initial perturbation of the system from its equilibrium. The latter provokes the system's natural response dynamics; e.g. when a pendulum is pulled back from its equilibrium state and released in a different initial state, its natural frequency can be determined by measuring the frequency it swings at. Since any externally applied excitations such as enforcing frequencies or an imposed frequency detuning [Schmidt et al., 1998] could hinder natural HHI, we decided not to actively control the interaction of the partners during our experiment, rather to observe it. Thus, the experiment can be classified as passive, which includes that parameters of the systems can not be changed and only bivariate data are available [see also Pikovsky et al., 2001]. With our experimental design, the idea is to trigger different initial phase differences $\Phi_{0}=\Phi(t=0)$ (c.f. Fig. 8). The dyad's natural response to the applied start off conditions in the experiment serves then for the identification of the parameter sets.

Note: If the frequency detuning $\Delta \omega$ within the interacting system is known, e.g. the eigenfrequencies of oscillatory mechanical systems can be controlled, the coupling strength $K$ could be alternatively identified from the phase difference $\Phi_{e}$ during equilibrium $\dot{\Phi}=0$ observed for different values of $\Delta \omega$ via the regression method of Schmidt et al. [1998]. 


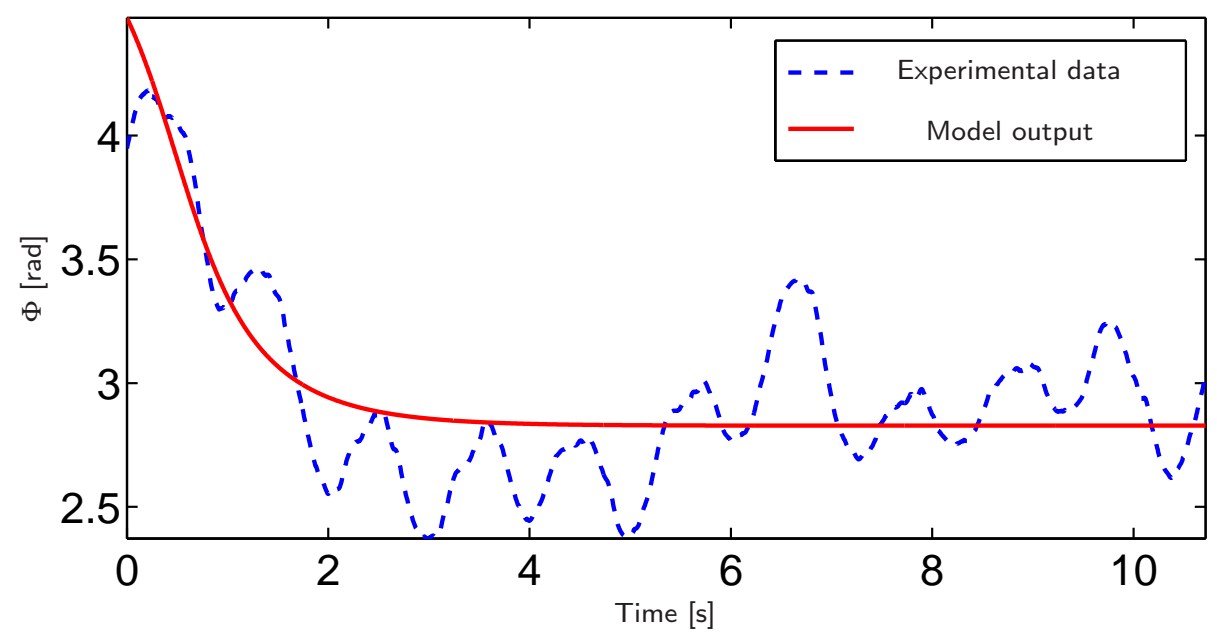

Figure 11: Comparison of the phase differences $\Phi(t)$ analytically derived with the spectral method and simulated with the parameterized model. The parameters $\left[\mathrm{rad} \cdot \mathrm{s}^{-1}\right]$ of the trial are $\Delta \omega=-0.49$ and $K=0.41$ at an RMSE $=0.23$

\subsubsection{Estimation of the model parameters}

The model structure which is given by the non-linear dynamical equation (24) and the time series of the phase difference $\Phi(t)$ enables an estimation of the parameters $(\Delta \omega, K)$ for each trial. The estimation problem is solved with the iterative predictionerror minimization method (PEM), which minimizes an error objective function depending on the simulated model output and the time series data [Ljung, 1999]. A so-called grey box model which implements (24) is fed into the PEM-algorithm of MATLAB's System Identification Toolbox and the initial state of the model is estimated jointly with the parameter set to achieve best fitting. Both the time-series data of the analytically derived phase difference and the simulated model output after the parameter estimation are illustrated for a representative trial in Fig. 11. The dynamics of the trial investigated here are judged to be stable according to (28), hence the simulated phase difference converges to the attractor point close to $\pi$. Possible reasons for the oscillatory phase fluctuations (c.f. Fig. 11) present in most of the trials are discussed in Sect. 5.

\subsubsection{Results of the parameter estimation}

For comparison, the model parameters estimated for phase difference time series acquired with the state-space, spectral and hybrid method are summarized in Table 1. Since the transformation of motion trajectories to phases is not unique but based on certain assumptions as introduced in Sec. 3.2, the applied method affects the modeling results and makes it part of the modeling approach. More than two thirds of the trials yield parameter sets with stable point attractors of the resulting dynamical system according to the stability criterion (28). Thus, it can be stated that the synchronization 
Table 1: Estimated model parameters, RMSE assessing model fit and number of trials $N_{\text {stable }}$ with stable parameter sets. Means are taken over $N_{\text {valid }}=294$ trials. SDs are taken over dyads. The results $\left[\mathrm{rad} \cdot \mathrm{s}^{-1}\right]$ are presented separately for the state-space, spectral and hybrid method

\begin{tabular}{cccccccc}
\hline Method & Mean $|K|$ & $\mathrm{SD}|K|$ & Mean $\left|K_{s}\right|$ & Mean $\Delta \omega$ & $\mathrm{SD} \Delta \omega$ & Mean RMSE & $N_{\text {stable }}$ \\
\hline State-space & 0.29 & 0.14 & 0.34 & -0.08 & 0.19 & 0.41 & 218 \\
Spectral & 0.20 & 0.05 & 0.24 & -0.06 & 0.14 & 0.26 & 223 \\
Hybrid & 0.29 & 0.11 & 0.33 & -0.09 & 0.15 & 0.59 & 202 \\
\hline
\end{tabular}

effects visible in Fig. 6 and 7 are reproduced by stable parameter sets in a majority of the trials.

For statistical analysis of the obtained results, one-way repeated measures ANOVAs with the within-subject factor method (spectral, hybrid, state-space) were performed. In order to compare the methods, data was averaged over start off conditions. GreenhouseGeisser correction was applied when the sphericity criterion was not met. The influence of the unstable parameter sets on the overall coupling gain is rather small; if only the trials are considered, in which the partners synchronize according to (28), the coupling gain (denoted with $\left|K_{S}\right|$ in Table 1) is not much higher than $|K|$. In both cases a significant main effect between methods is observed, $|K|: F(2,18)=3.87, p<.05$, $\left|K_{S}\right|: F(2,18)=3.88, p<.05$. Contrasts show that the spectral method results in the smallest coupling gain on average compared to both the hybrid, $|K|: F(1,9)=8.37$, $p<.05,\left|K_{S}\right|: F(1,9)=6.09, p<.05$, and the state-space method $|K|: F(1,9)=7.15$, $p<.05,\left|K_{s}\right|: F(1,9)=7.50, p<.05$, with the latter not being different from each other. This illustrates that the commonly detected synchronization effects are explained by a non-zero coupling term, though no instructions are given which actively modulate the interaction in the experiment.

A mean $\Delta \omega$ close to zero indicates a rather balanced frequency detuning over all dyads. No significant differences between methods are found, $p>$.6. However, a small negative component of $\Delta \omega$ points to the trend that the individual cycle frequency preferred by person 2 is on average slightly higher than person 1 's frequency. The considerably high SD $\Delta \omega$ leads to the conclusion that the frequency detuning predicted by the model is an individually varying parameter.

\subsubsection{Evaluation of the model fit}

For comparison of the root-mean-square-error of model prediction and measurement (RMSE in Table 1) a one-way repeated measures ANOVA with the within-subject factor method (spectral, hybrid, state-space) was performed. Degrees of freedom were corrected with the Greenhouse-Geisser method. Here, highly significant differences between methods are observed, $F(1.1,9.7)=28.37, p<.001$. Best model fit is achieved by the combination of the extended Kuramoto model and the spectral method. It differs significantly from the RMSE obtained with the state-space method, $F(1,9)=167.47, p<.001$, or the hybrid method, $F(1,9)=38.53, p<.001$. Furthermore, RMSE is lower with the 
Table 2: Results of the trial-wise regression: $R^{2}$ represents the percentage of the variance explained by the model, $N_{\text {valid }}$ is the number of valid trials which were included into analysis for the respective condition and $N_{p<.001}$ list how many times the model fit is above chance (at $p<0.001$ )

\begin{tabular}{ccccc}
\hline Method & Condition & Median $R^{2}$ & $N_{\text {valid }}$ & $N_{p<.001}$ \\
\hline State-space & zero-cycle & 0.313 & 98 & 98 \\
& quarter-cycle & 0.994 & 97 & 97 \\
& half-cycle & 0.996 & 99 & 99 \\
\hline \multirow{2}{*}{ Spectral } & zero-cycle & 0.350 & 98 & 97 \\
& quarter-cycle & 0.971 & 97 & 96 \\
& half-cycle & 0.998 & 99 & 99 \\
\hline Hybrid & zero-cycle & 0.223 & 98 & 98 \\
& quarter-cycle & 0.904 & 97 & 97 \\
& half-cycle & 0.995 & 99 & 99 \\
\hline
\end{tabular}

state-space than with the hybrid approach, $F(1,9)=11.20, p<.01$. This is due to the filtering property of the Hilbert transform, as the frequency at the maximum of the power spectrum determines the instantaneous frequency and phase respectively. Both the state-space and hybrid method are applied without any filtering technique and thus yield a higher phase variability than the spectral method, which is not explained by the model.

Additionally, the model's goodness of fit in combination with the three phase estimation methods is assessed with the R-square metric $\left(R^{2}\right)$, which allows for an estimation of how much variance of the data (in \%) are explained by the respective model. The median values of the trial-wise $R^{2}$ are summarized in Table 2 . In order to find out how often the variance of the data is explained above chance level, the significance of each trial's model fit is tested by calculating the F-ratio. The numbers of significant fits are reported in Table 2 .

\section{Discussion}

Both the results from data analysis and model synthesis provide evidence for and characterize the emerging synchronization of movements. Treating human dyadic interaction in a repetitive, goal-directed task as a synchronization problem with the phase difference of coupled oscillators as the collective seems to be a valid approach. Its implications are discussed in the following.

\subsection{Performance comparison of the phase transformations}

Three different methods on the calculation of instantaneous phases from experimental movement data are evaluated in this study. Their performance is assessed 1) by look- 
ing at the synchronization index, which penalizes the phase variability and 2) by the RMSE, which accounts for the residuals after model identification. When comparing the values of the synchronization index and the RMSE-based model fit, both measures yield similar performance trends between the methods on phase transformation. This similarity can be explained by the following fact: the proposed model structure replicates the dominant process of phase convergence only, and therefore in the model residuals any additional variance of the collective becomes directly visible, which affects both the RMSE and the synchronization index in the same way. Best performance results are achieved when the phases are generated by the spectral method. While it is a powerful tool when it comes to off-line analysis, the spectral method is less suitable for on-line application, since a short-time implementation of the Hilbert transform would be required, at the cost of signal delay. For movement tasks comprised by a quasi-harmonic action sequence, where a state-space can be defined and its state variables can be measured, the state-space method is a good alternative, which can be also deployed on-line. The concept of the hybrid method is superior to both the state-space and the spectral method, if the phase of arbitrary complex, non-harmonic primitive sequences is to be estimated on-line. When applied to the experimental data set, the performance of the hybrid method is inferior to the state-space and the spectral method in its current implementation. It has to be remarked that task knowledge is encoded by the mathematical representations (8) and their transitions induced by the segmentation. Both are not unique but affect the resulting performance of the approach. There are other representations than the minimum jerk model introduced as an approximation of the movement primitives in our experiment: e.g. for perfect harmonic movements, results equivalent to the state-space method could be obtained when the action sequence is segmented into two movement primitives, each represented by a trigonometric function. Enhancing the segmentation of the action sequence by predictive parameterizations as well as improving the primitives' mathematical representations by estimation techniques with subsequent evaluations of the hybrid method in complex movement tasks is a promising way to extend the power of the coordination model.

\subsection{Interpretation of the model residuals}

The extended Kuramoto model predicts only the dominant component of the collective's dynamics by a first-order non-linear dynamical system. The median $R^{2}$ under condition zero-cycle is remarkably low regardless of the method. Since the measured phase difference remains constantly close to the attractor $\Phi=0$ for most of the zerocycle trials, the explanatory power of the extended Kuramoto model in these trials does not exceed much that of the pure mean value of the measured phase difference. Potential additional information content might be present in the model residuals. Through a spectral analysis of the residuals, oscillatory components at frequencies close to the participants' cycle frequencies ( $\mathrm{M}=0.73 \mathrm{~Hz}$ over all trials) can be discovered in many of the trials (c.f. Fig. 11), which is in line with the observations made by Schmidt et al. [1993] during pendulum swinging. These oscillations can be explained by the following reasons.

First and foremost, the observed phase fluctuations can be artifacts of the phase estimation. The instantaneous phase of arbitrary oscillatory time series such as human 
movement trajectories can be uniquely determined only over cycles for a discrete event in the cycle, while the evolution within cycles strongly depends on the reconstruction method. Neither the movement trajectories of the individuals are purely harmonic nor the phase estimation techniques achieve perfect harmonic phases. Thus, the state-space plots are not perfectly circular but slightly distorted, see Fig. 4. Even if we assume equally distorted state-space plots for both partners, the phase difference $\Phi(t)$ will oscillate at multiples of the cycle frequency for the case $\Phi(t) \neq 0$. In their note on coordination models, Fuchs and Kelso [1994] show that these phase fluctuations can be reproduced by the original $\mathrm{HKB}$ model comprised by non-harmonic oscillators. Within our modeling approach, this effect can be accounted for by further development of the phase estimation techniques with better harmonicity, which is also a prerequisite if more complex goal-directed tasks should be addressed.

Second, the coupling strength between the agents might not be constant over time but rather include components depending on the individual phase. This assumption is backed by the workspace topology of the experimental task investigated here. Since simultaneous visual attention to one's own and the opponent's movement is easier when getting close to the target area and the partner's workspace respectively, it might effect a stronger coupling strength. The integrative role of the visual perception of relative phase has been shown by Wilson et al. [2005]; it affects the stability of coordination.

\subsection{Interpretation of the modeling results}

In most of the trials, the participants fall into rhythmic patterns to synchronize as the effect of an emerging joint behavioral goal, although precision is required as individual goals for the partners. Since experimental results might be strongly task-dependent, we put special emphasis on the design of the task paradigm. The experimental setting is natural in the sense of bearing similarity to repetitive, goal-oriented action tasks. People are not instructed to synchronize, but only get an instruction required for the individual task performance, which allowed natural interaction to emerge. Besides the start off condition, we did not introduce any control variables that artificially modulate the flow of interaction. This should allow an interpretation of the results in the light of natural HHI.

As a result of the system identification, values of the parameter set $(\Delta \omega, K)$ are found per trial. Within our modeling approach, the parameters are assumed to be timeinvariant on a short time scale, i.e. over the course of one experimental trial. The parameter sets averaged over trials can be treated as behavioral features of the dyads. However, accidental as well as deliberate parameter variations might be present due to both environmental influences and intrinsic changes of human behavior.

The frequency detuning $\Delta \omega$ expresses shifts of the equilibrium points to which the collective is attracted. It can serve as a measure of discrepancy regarding the agents' individually desired cycle frequency in the joint action task. Furthermore, hypothetical natural frequencies of the agents can be determined. Their meaning might extend to the self-organization of leader and follower roles during movement coordination, which deserves further investigation in the light of role behavior among the agents.

The coupling gain $K$ quantifies a dyad's weighing of two potentially competing goals: just being precise to fulfill the instructed goal versus being synchronized with 
the partner as an additional, voluntary goal. Non-zero mean values of $K$ averaged over all trials indicate emerging interaction between the partners which leads to the observed patterns of synchronization. The regression model employed by Schmidt et al. [1998] to measure coupling strength and the extended Kuramoto model proposed in this paper are locally identical, hence the resulting coupling from both task paradigms can be related to each other. The overall mean ${ }^{3}$ of $2|K|\left(0.52 \mathrm{rad} \cdot \mathrm{s}^{-1}\right)$ in Table 1 is about one third of the mean value $\left(1.70 \mathrm{rad} \cdot \mathrm{s}^{-1}\right)$ reported by Schmidt et al. [1998] for the local model coupling strength of (intentional) interpersonal coordination. Since in their experiments, the participants were instructed to swing pendulums either in in-phase or anti-phase relation, a coupling stronger than in our setting could be expected.

\section{Conclusion}

In this article we present a step-wise approach to a model of inter-human movement coordination. Motion trajectories were recorded in a novel HHI-experiment which successfully integrates repetitive and goal-directed action. Synchronization is found to be an essential principle of human movement coordination during goal-directed action. The human dyads which participated in our study synchronized their movements. Governed by a dynamical process they fell into in-phase as well as anti-phase relations for most of the trials. The emerging relations are successfully replicated by the attractor dynamics of coupled phase oscillators inspired by the Kuramoto model, which is an oscillator model described by the evolution of its phases. Three different methods on transforming the movement trajectories into instantaneous phases are investigated; closest fitting between experimental data and the model is achieved by the spectral method, which is well-suited only for the off-line analysis of simple repetitive actions. While the state-space method extends the phase estimation to on-line application, the concept of a novel hybrid method is introduced, which allows to derive instantaneous phases for arbitrary complex action sequences. Using a technique that does not influence natural HHI, system identification is performed to estimate the model parameters, which are the coupling strength and the frequency detuning among the dyad. Stable attractor points resulting from the identified model match the relations observed in the experimental data.

The presented approach based on coupled phase dynamics facilitates the modeling of the partners' interactive behavior even when they are engaged in heterogeneous action tasks. The identified model can be readily used to generate the actions of a robotic agent on-line. In a follow-up study we will deploy the model to an anthropomorphic robot, in order to answer the question: Does a model of inter-human movement coordination enhance human-robot interaction?

\footnotetext{
${ }^{3}$ Values of $K$ have to be doubled for comparison, since $K$ refers to the single agent's unilateral coupling in our work.
} 


\section{Acknowledgements}

The authors would like to thank Lars Kreutz for his support in setting up and conducting the experiment. This work is supported in part within the DFG excellence initiative research cluster Cognition for Technical Systems - CoTeSys (www . cotesys . org).

\section{Experiment}

\section{Participants}

In total 20 people (13 male, 7 female) participated in the experiment forming 10 dyads. They were between 18 and 28 years old $(M=23.5)$. All were right handed, had normal or corrected-to-normal vision and were naïve as to the purpose of the experiment. For participation they were paid $8 €$ per hour.

\section{Experimental Setup}

Participants were sitting face to face at a round table on which four circles were marked in two different colors, see Fig. 12. Each color was assigned to one person who was equipped with a marking pen of equal type and size in the respective color. Participants had to hold the pens in their right hands forming a fist around them with the thumb pointing upwards. With this it was achieved that the pen was always in a orthogonal relation to the table surface. We encouraged participants to sit in an upright position and instructed them to put the left hand on their lap. To reduce tapping sound, pieces of felt were attached to the pen tips.

During task performance, participants' hand movements were captured with an infrared 3D-motion tracking system (PTI VisualEyez II VZ4000) at a sampling rate of $30 \mathrm{~Hz}$ for acoustical signal triggering and at a sampling rate of $200 \mathrm{~Hz}$ for data analysis. LED markers used for motion capturing were attached to the top end of the pen and to the participants' basis thumb joint. Both participant had to wear a pair of stereo phones (SONY MDR-XD200) used to present an individual acoustic trigger signal. For being able to review task performance later, hand movements were additionally recorded by a video camera.

\section{Procedure and Design}

The experiment started with capturing the individual calibration positions for each dyad. For this purpose participants had to put their pen to their individual start and target point one time respectively. The written instruction included a description of the task which was to alternately tap the individual start and target point with the pen tip. Furthermore participants were asked to carry the pen from one point to the other. Sliding the pen over the table was not allowed. No instructions were given regarding speed in order to provoke natural behavior.

At the beginning of each trial participants were asked to rest in their respective start position and instructed to start executing the task as soon as they heard the acoustical 


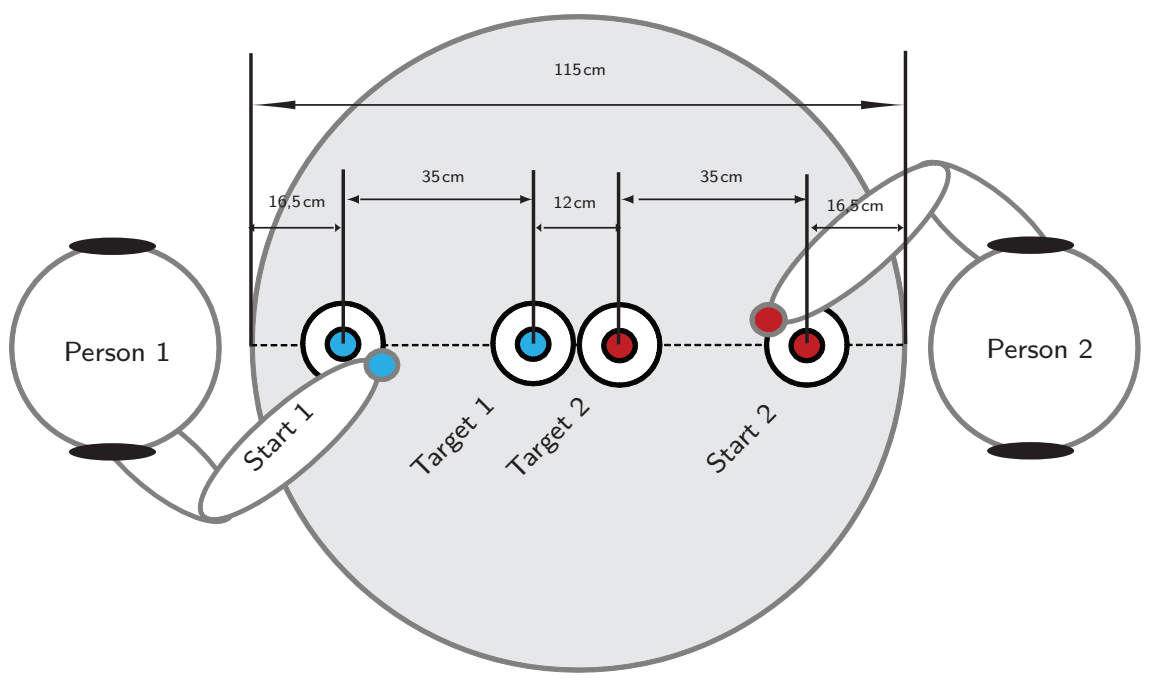

Figure 12: Experimental setup with dimensions. Two agents denoted as person 1 and person 2 performed identical motor tasks while sitting in chairs and facing each other. The task was to alternately tap on two assigned dots (start and target, diameter $8 \mathrm{~mm}$ ) with a pen in hand. The dots were marked on a round table and each one was surrounded by a white area (diameter $60 \mathrm{~mm}$ )

start signal (high-pitched tone) through their phones. Simultaneously with the start signal, motion capturing started. The stop signal(low-pitched tone) was presented automatically after both participants had performed at least 10 cycles each. At the same time, motion capturing stopped and participants had to move their pen back to the start point.

Three start off conditions were applied which provoked differing spatial relations: 1) the start signals for both participants were presented simultaneously (zero-cycle), 2) the start signal for the second person was presented when the first person has already made half the distance between the start and the target point for the first time (quartercycle), 3) the start signal for the second person was presented when the first person had reached the target point for the first time (half-cycle). Six sets each consisting of six trials were performed which led to a total of 36 trials. Start off conditions were kept constant within sets which led to a total of 12 trials per condition. In the conditions quarter-cycle and half-cycle, the delay of the start signal for the second person was calculated on-line from the movement data of the first person. Being first person was randomly assigned to person 1 or person 2 and counterbalanced in each set. The first trial in each set was excluded from analysis. 


\section{References}

P. Beek, R. Schmidt, A. Morris, M. Sim, and M. Turvey. Linear and nonlinear stiffness and friction in biological rhythmic movements. Biol. Cybern., 73:499-507, 1995a. ISSN 0340-1200.

P J Beek, C E Peper, and D F Stegeman. Dynamical models of movement coordination. Hum. Mov. Sci., 14:573-608, 1995 b.

P J Beek, C E Peper, and A Daffertshofer. Modeling Rhythmic Interlimb Coordination: Beyond the Haken-Kelso-Bunz Model. Brain Cogn., 48(1):149-165, 2002. ISSN 0278-2626.

Andreas Bruns. Fourier-, Hilbert- and wavelet-based signal analysis: are they really different approaches? J. Neurosci. Methods, 137(2):321-332, 2004. ISSN 01650270 .

Avis H. Cohen, Philip J. Holmes, and Richard H. Rand. The nature of the coupling between segmental oscillators of the lamprey spinal generator for locomotion: A mathematical model. J. Math. Biol., 13:345-369, 1982. ISSN 0303-6812.

Aymar de Rugy, Robin Salesse, Olivier Oullier, and Jean-Jacques Temprado. A neuromechanical model for interpersonal coordination. Biol. Cybern., 94(6):427-443, 2006.

T. Eisenhammer, A. Hübler, N. Packard, and J. A. S. Kelso. Modeling experimental time series with ordinary differential equations. Biol. Cybern., 65:107-112, 1991. ISSN 0340-1200.

T Flash and N Hogan. The coordination of arm movements: An experimentally confirmed mathematical model. Neurosci., 5:1688-1703, 1985.

Armin Fuchs and J. A. Scott Kelso. A theoretical note on models of interlimb coordination. J. Exp. Psychol. Hum. Percept. Perf., 20:1088-1097, 1994.

H Haken, J A S Kelso, and H Bunz. A theoretical model of phase transitions in human hand movements. Biol. Cybern., 51(5):347-356, 1985.

V K Jirsa and J A S Kelso. The excitator as a minimal model for the coordination dynamics of discrete and rhythmic movement generation. J. Mot. Behav., 37:35-51, 2005.

B. A. Kay, J. A. S. Kelso, E. L. Saltzman, and G. Schöner. Space-time behavior of single and bimanual rhythmical movements: data and limit cycle model. J. Exp. Psychol. Hum. Percept. Perform., 12:178-192, 1987.

Thomas Kreuz, Florian Mormann, Ralph G Andrzejak, Alexander Kraskov, Klaus Lehnertz, and Peter Grassberger. Measuring synchronization in coupled model systems: A comparison of different approaches. Phys. D: Nonlinear Phenom., 225(1): 29-42, 2007. ISSN 0167-2789. 
Y Kuramoto. Chemical Oscillations, Waves and Turbulence. Springer, Heidelb., 1984.

L Ljung. System identification: Theory for the user. Prentice Hall, Upper Saddle River, 1999.

T Lorenz, A Mörtl, B Vlaskamp, A Schubö, and S Hirche. Synchronization in a goaldirected task: Synchronization in a goal-directed task: human movement coordination with each other and robotic partners. In Proc. IEEE Ro-Man, 2011.

Lynden K Miles, Louise K Nind, and C Neil Macrae. The rhythm of rapport: Interpersonal synchrony and social perception. J. Exp. Soc. Psychol., 45(3):585-589, 2009. ISSN 00221031.

A Pikovsky, M Rosenblum, and J Kurths. Synchronization - A universal concept in nonlinear sciences, pages 153-172. Cambridge University Press, 2001.

R H Rand, A H Cohen, and P J Holmes. Neural Control of Rythmic Movement in Vertebrates, chapter Systems of Coupled Oscillators as Models of Central Pattern Generators. Wiley, New York, 1988.

Michael J Richardson, Kerry L Marsh, and R C Schmidt. Effects of visual and verbal interaction on unintentional interpersonal coordination. J. Exp. Psychol. Hum. Percept. Perform., 31(1):62-79, 2005. ISSN 0096-1523.

Michael J Richardson, Kerry L Marsh, Robert W Isenhower, Justin R L Goodman, and R C Schmidt. Rocking together: Dynamics of intentional and unintentional interpersonal coordination. Hum. Mov. Sci., 26(6):867-891, 2007. ISSN 0167-9457.

M Rosenblum and J Kurths. Nonlinear Analysis of Physiological Data, chapter Analysing Synchronization Phenomena from Bivariate Data by Means of the Hilbert Transform, pages 91-99. Springer, Heidelb., 2007.

R C Schmidt and Beth O'Brien. Evaluating the Dynamics of Unintended Interpersonal Coordination. Ecol. Psychol., 9(3):189-206, 1997.

R C Schmidt, C Carello, and M T Turvey. Phase transitions and critical fluctuations in the visual coordination of rhythmic movements between people. J. Exp. Psychol. Hum. Percept. Perform., 16(2):227-247, 1990. ISSN 0096-1523.

R C Schmidt, B K Shaw, and M T Turvey. Coupling Dynamics in Interlimb Coordination. J. Exp. Psychol. Hum. Percept. Perform., 19(2):397-415, 1993.

R C Schmidt, M Bienvenu, P A Fitzpatrick, and P G Amazeen. A comparison of intra- and interpersonal interlimb coordination: coordination breakdowns and coupling strength. J. Exp. Psychol. Hum. Percept. Perform., 24(3):884-900, June 1998. ISSN 0096-1523.

G Schöner and J A Kelso. Dynamic pattern generation in behavioral and neural systems. Sci., 239(4847):1513-1520, 1988. 
Dmitry A Smirnov and Ralph G Andrzejak. Detection of weak directional coupling: Phase-dynamics approach versus state-space approach. Phys. Rev. E, 71(3):36207, 2005.

M T Turvey, Lawrence D Rosenblum, R C Schmidt, and Peter N Kugler. Fluctuations and Phase Symmetry in Coordinated Rhythmic Movements. J. Exp. Psychol. Hum. Percept. Perform., 12(4):564-583, 1986.

Piercarlo Valdesolo, Jennifer Ouyang, and David DeSteno. The rhythm of joint action: Synchrony promotes cooperative ability. J. Exp. Soc. Psychol., 46(4):693-695, 2010 .

Niek R van Ulzen, Claudine J C Lamoth, Andreas Daffertshofer, Gün R Semin, and Peter J Beek. Characteristics of instructed and uninstructed interpersonal coordination while walking side-by-side. Neurosci. Lett., 432(2):88-93, 2008. ISSN 0304-3940.

Manuel Varlet and Michael J Richardson. Computation of continuous relative phase and modulation of frequency of human movement. J. Biomech., 44(6):1200-4, April 2011. ISSN 1873-2380.

Cordula Vesper, Alexander Soutschek, and Anna Schubö. Motion coordination affects movement parameters in a joint pick-and-place task. Q. J. Exp. Psychol., 62(12): 2418-2432, 2009.

William H Warren. The Dynamics of Perception and Action. Psychol. Rev., 113(2): 358-389, 2006. ISSN 0033-295X.

Andrew D Wilson, David R Collins, and Geoffrey P Bingham. Perceptual coupling in rhythmic movement coordination: stable perception leads to stable action. Exp. Brain Res., 164(4):517-528, 2005. ISSN 0014-4819. 